\title{
Sea-ice microbial communities in the Ross Sea: autumn and summer biota
}

\author{
David L. Garrison ${ }^{1,2, *}$, Angela Gibson ${ }^{1}$, Susan L. Coale ${ }^{1}$, Marcia M. Gowing ${ }^{1}$, \\ Yuri B. Okolodkov ${ }^{3}$, Christian H. Fritsen ${ }^{4}$, Martin O. Jeffries ${ }^{5}$ \\ ${ }^{1}$ Institute of Marine Sciences, University of California Santa Cruz, Santa Cruz, California 95064, USA \\ ${ }^{2}$ National Science Foundation, Division of Ocean Sciences, Biological Oceanography Program, Room 725, \\ 4201 Wilson Blvd., Arlington, Virginia 22230, USA \\ ${ }^{3}$ Centro de Ecología y Pesquerías, Universidad Veracruzana, Calle Hidalgo No. 617, Col. Río Jamapa, Boca del Río, \\ Veracruz, CP 94290, Mexico \\ ${ }^{4}$ Division of Earth and Ecosystem Sciences, Desert Research Institute, 2215 Raggio Parkway, Reno, Nevada 89512, USA \\ ${ }^{5}$ Geophysical Institute, University of Alaska Fairbanks, Fairbanks, Alaska 99775-7320, USA
}

\begin{abstract}
The composition of sea ice communities in the Ross Sea region was examined during the autumn to winter transition and during the summer. The biomass of autotrophs and heterotrophs in autumn reached maximum values of 709 and $167 \mathrm{mg} \mathrm{C} \mathrm{m}^{-2}$, and in summer maximum values of 3873 and $209 \mathrm{mg} \mathrm{C} \mathrm{m}^{-2}$, respectively. During the autumn-winter cruise, most of the biomass was found within ice floes as interior and bottom layer communities. During summer, surface-layer slush communities occurred throughout the ice-covered regions. The biomass was highly variable throughout the study regions during both cruises. Diatoms dominated the autotrophic biomass; however, autotrophic dinoflagellates and autotrophic flagellates contributed significantly to the community make-up. Among heterotrophs, ciliates predominated during both cruises, followed by heterotrophic flagellates and heterotrophic dinoflagellates. Similarity analysis, based on the biomass composition of major groups, showed consistency between and within cruises, with most samples $>70 \%$ similar. The autumn to winter samples (all from within floes) showed higher similarity clusters that could be related to changing compositions of diatoms, ciliates, and autotrophic dinoflagellates. Most variable were some summer surface slush samples, where samples dominated by Phaeocystis, Pyramimonas, Gymnodinium, and the ciliate Gymnozoum formed outlying clusters. The dynamics of ice biota may be determined by relatively few taxa that are persistently found within the ice floes. Surface blooms may develop either from a biota within the ice or from opportunistic forms from the water column that are introduced during flooding events. Thus, these assemblages may show considerably more variability in composition than those that develop within the underlying ice.
\end{abstract}

KEY WORDS: Sea ice community $\cdot$ Antarctic $\cdot$ Protists

Resale or republication not permitted without written consent of the publisher

\section{INTRODUCTION}

Ice-associated biotic communities are conspicuous in the vast areas of drifting pack ice surrounding Antarctica (reviewed by Garrison 1991, Palmisano \& Garrison 1993, and Garrison \& Mathot 1996). Sea-ice communities are most often characterized by measurements of biomass proxies such as chlorophyll a (chl a) and particulate organic carbon and nitrogen (POC and PON) (e.g. Dieckmann et al. 1998), and relatively few studies have evaluated the community structure in terms of the species or the major taxa present (Garrison 1991, Garrison \& Mathot 1996). Early descriptions of the Antarctic ice biota emphasized the predominance of diatoms (e.g. Horner 1985a,b). However, it has become apparent from more recent studies (those using methods suitable for preserving and distinguishing a larger variety of microorganisms and determining their 
trophic status) that a more diverse assemblage of autotrophs and heterotrophs inhabits the sea ice (Garrison \& Buck 1985, 1989, 1991, Garrison \& Mathot 1996).

Relatively few studies have been sufficiently comprehensive to characterize the biomass composition (but see Garrison \& Close 1993), and the degree to which the species are known and described varies tremendously within the major groups present. Although there have been detailed reports of some icemicrobial groups (e.g. diatoms: Garrison et al. 1987), there are other groups, often occurring at ecologically significant abundances, where the species are still largely unknown (Thomsen et al. 1991). Several 'new' or rare organisms have been described from the ice habitat (Mitchell \& Silver 1982, Corliss \& Snyder 1986, Dahms \& Dieckmann 1987, Buck et al. 1990a,b, 1992, Thomsen et al. 1991, 1997, Stoecker et al. 1992, 1993, Daubjerg et al. 2000). It is still not clear whether these findings indicate that ice is a unique habitat (see Thomsen et al. 1997) or whether unusual and rarer organisms are just easier to discover in the ice where populations are often highly concentrated. Molecular approaches have been applied to the study of the ice biota (Montresor et al. 1999, 2003, Gast et al. 2001, 2002, 2003), but to date, the most detailed descriptions are still based on microscopy methods.

There is increasing evidence that the sea-ice biota has an ecologically important role in the Antarctic icecovered regions. Sea ice is a site of primary production with a seasonal pattern of production and biomass accumulation that both extends and increases regional productivity (e.g. Legendre et al. 1992, Lizotte 2001). Production and accumulation of biomass in ice, including both autotrophic and heterotrophic forms and probably detritus, is an important seasonal resource for some ecologically important species such as Euphausia superba (Daly 1990). Observations of a diverse microbial assemblage in ice (cited above and see review by Garrison \& Mathot 1996) have suggested that the ice habitat is similar to that of the water column with respect to an active microbial food web. Recent experimental work (Maranger et al. 1994, Laurion et al. 1995, Sime-Ngando et al. 1997, 1999) has confirmed this prediction. Observational evidence (Garrison et al. 1987, Lizotte 2001) supports the proposition that pelagic bloom dynamics can also be influenced by 'seeding' from the ice. A full understanding of the biological community dynamics within sea ice clearly requires information beyond proxy measurements of biomass alone (e.g. such as chl a measurements). Our studies of ice communities in the Ross Sea have included the consideration of a range of microbial forms from viruses (Gowing et al. 2002, 2004, Gowing 2003) and bacteria (Stewart et al. 2001, Gowing et al.
2004) to protists. Here, we describe the community composition of the ice biota in pack ice regions of the Ross Sea during the autumn to winter transition and during the summer. The present analyses have been restricted to the protists in ice to take advantage of a large number of samples where we have comparable data for this component of the microbial community.

\section{MATERIALS AND METHODS}

Samples were collected from the pack ice regions of the Ross Sea during the autumn of 1998 (NBP98-3) and the summer of 1999 (NBP99-1) (Fig. 1). Sea ice was sampled at a number of stations to characterize the ice crystal texture and modes of formation (Jeffries et al. 2001), and at many of these stations community biomass parameters such as chl $a$, and POC and PON were measured (Fritsen et al. 2001, Garrison et al. 2003). To provide a more complete description of the biological assemblages in ice, 10 stations from NBP983 (autumn) and 13 stations from NBP99-1 (summer) were analyzed using epifluorescence and bright-field light microscopy techniques (see Fig. 1 for station locations).

At ice floe sampling stations, snow depth and ice thickness were measured along $150 \mathrm{~m}$ transects. Then, at 3 locations selected to be representative of the snow and ice variability of the transect, a $7.5 \mathrm{~cm}$ diameter ice

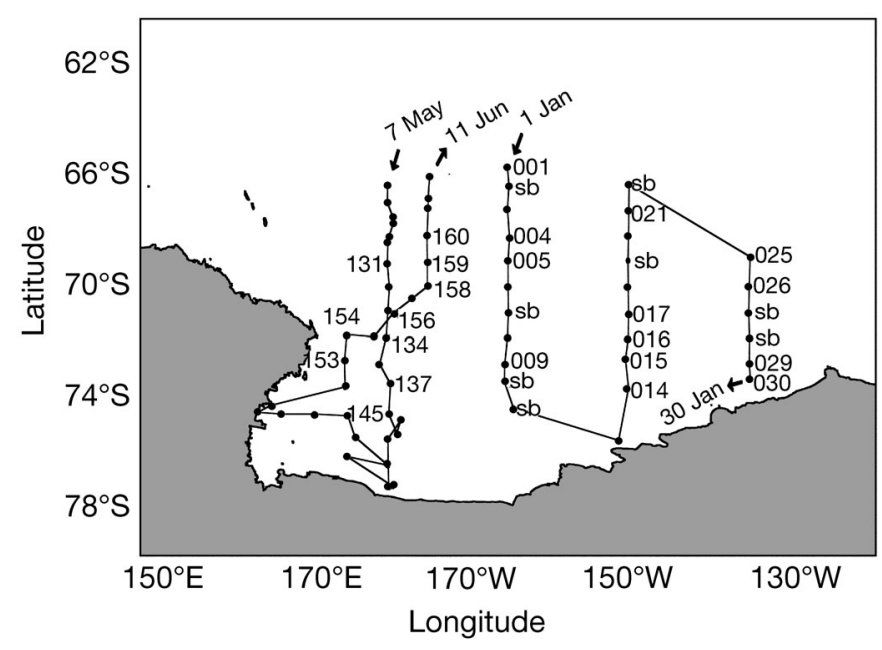

Fig. 1. Ross Sea Region. Cruise track of the 'Nathaniel B. Palmer' in the Ross Sea showing ice stations (filled circles) sampled during cruise NBP98-3 (Stns 131-160; 7 May to 11 June 1998) and NBP99-1 (Stns 001-030; 1 to 30 January 1999). Stations where ice cores were collected for biota determinations are identified by day of the year. During NBP99-1 stations identified as 'sb' are where only surface slush was collected. Other stations identified, except 014 and 030, had both cores and surface slush collections 
coring auger was used to obtain 2 ice cores for physical analysis. At one of the locations, an ice core was taken for biological analysis. One of the physical ice cores was processed on site; ice temperatures were measured at $10 \mathrm{~cm}$ intervals along the length of the core, which was then cut into $10 \mathrm{~cm}$ sections that were placed in plastic buckets and allowed to melt subsequently aboard the ship. The salinity of the melted samples was then measured. The second physical core was returned to the ship for ice crystal texture characterization to distinguish between granular and columnar ice; granular frazil ice and granular snow were subsequently distinguished from each other using stable oxygen isotopes (Jeffries et al. 2001).

The biology core was obtained within $50 \mathrm{~cm}$ of the physical cores. The total length was measured on site, and it was then cut immediately into $\sim 10$ to $20 \mathrm{~cm} \mathrm{sec-}$ tions (depending on total core length), which were stored in 41 wide mouth plastic jars. The jars were then returned to the ship and the ice samples allowed to melt in measured volumes (usually 1500 to $2000 \mathrm{ml}$ ) of filtered (pore size $0.2 \mu \mathrm{m}$ ) seawater. The dilution procedure is a precaution to protect delicate organisms from osmotic changes during melting (Garrison \& Buck 1986). The cores were allowed to melt for approximately $48 \mathrm{~h}$ at approximately $3^{\circ} \mathrm{C}$ in a cold room aboard ship. The original volume of the ice was determined by subtracting the added seawater volume from the final volume, and dilution factors were calculated to correct organism abundance estimates.

Parameters measured from cores collected to characterize the physical stratigraphy and physical environment in the ice included in situ temperatures at $10 \mathrm{~cm}$ intervals throughout ice cores, bulk salinities measured on melted core sections, and the structural make-up of the ice core as determined from examining thin vertical sections between cross-polarized filters to distinguish ice structural types (Jeffries et al. 2001). In addition, samples of these cores were retained for stable oxygen isotope analysis to aid in the distinction between granular frazil ice and granular infiltration ice (snow ice) layers.

Aliquots (400 to $800 \mathrm{ml}$ ) of the diluted, melted ice samples were filtered through $25 \mathrm{~mm}$ Whatmann glass fiber filters (GF/F) at $\leq 7$ psi for the analysis of chl $a$. Ten $\mathrm{ml}$ of $90 \%$ acetone was added to the GF/f filter and allowed to extract pigments for $\sim 24 \mathrm{~h}$ (no less than $4 \mathrm{~h}$ ) at $-20^{\circ} \mathrm{C}$ in the dark. Extracts were then allowed to warm to room temperature (in the dark) and were centrifuged for $5 \mathrm{~min}$ (to settle any filter particles). The fluorescence of extracted pigments was measured both before and after acidification $(10 \% \mathrm{HCl})$ using a Turner Designs fluorometer calibrated with pure chl a (Sigma). Chlorophyll concentrations were calculated as described in Parsons et al. (1984), with a correction for the dilution of the ice meltwater with filtered seawater.

Aliquots of ice melt were concentrated on polycarbonate filters for examination by epifluorescence microscopy, and additional aliquots were preserved for settling, identifying, and enumerating organisms using an inverted microscope (also using epifluorescence for some examinations). Using this combination of microscopy procedures, we were able to identify, count, and size a variety of organisms in the sea-ice assemblage, ranging from nano- through micro-sized organisms (Table 1).

Filter-concentrated samples for epifluorescence microscopy were prepared by passing 10 to $500 \mathrm{ml}$ of sample (separately, not in sequence) through 0.8 and $5.0 \mu \mathrm{m} 25 \mathrm{~mm}$ Nucleopore filters in a vacuum $\leq 7 \mathrm{psi}$. Prior to filtration, large aliquots of melted, diluted ice samples were preserved with glutaraldehyde $(\sim 1 \%$ final concentration for $1 \mathrm{~h}$ ). One $0.8 \mu \mathrm{m}$ filter was stained with 4',6-Diamidino-2-phenylindole (DAPI) (final concentration $3.5 \mu \mathrm{g} \mathrm{ml}^{-1}$ ) (Coleman 1980), and a replicate preparation was stained with Acridine Orange (AO) (final concentration $4.5 \mu \mathrm{g} \mathrm{ml}^{-1}$ ) (Hobbie et al. 1977). The $5.0 \mu \mathrm{m}$ filter was also stained with DAPI; volumes filtered with the $5.0 \mu \mathrm{m}$ filter were generally larger than for the smaller pore size filters because this preparation was intended for examining larger and rarer forms. Filters were mounted on microscope slides with Resolve low viscosity immersion oil and stored at $-80^{\circ} \mathrm{C}$ on the ship, transferred to our laboratory on dry ice, and there again stored at $-80^{\circ} \mathrm{C}$ until examined. Volumes concentrated on filters depended on the density and size of the organisms present; this was determined by examining filters at sea and altering volumes accordingly. At sea, filters were examined using a Zeiss Axioscope epifluorescence microscope, and in our laboratory, they were examined with an Olympus BX60 microscope. AOstained preparations were examined with blue excitation using a wideband exciter filter (band pass 450 to $480 \mathrm{~nm}$ ) and long pass filter (515 nm). The DAPIstained preparations were examined using UV excitation (band pass 330 to $386 \mathrm{~nm}$ ) and a long pass filter (420 nm).

Aliquots of $100 \mathrm{ml}$ were preserved with Lugol's iodine, and replicates were also preserved with $\sim 1 \%$ glutaraldehyde or $2 \%$ paraformaldehyde. These were settled using the Utermöhl settling technique to count large cells such as diatoms, dinoflagellates and ciliates with either an Olympus IMT-2 or an Olympus IX70 inverted microscope (see Garrison et al. 1998).

The abundance and biomass were determined in a similar manner for both filter-concentrated and settled samples. Abundance was estimated by examining parts or all of either filters or counting chambers. 
Table 1. Abundance and biomass of organisms in sea-ice communities from the Ross Sea pack ice region in Autumn (NBP98-3) and Summer (NBP99-1). Average and range (in parentheses) are shown for major groups; only ranges are given for species or lower taxonomic listings. Where a single value is shown for ranges, the taxon was found in only 1 sample. Major taxa used for community analysis: AFL: autotrophic flagellates; PHAEO: Phaeocytis; ADINO: autotrophic dinoflagellates; DIAT: diatoms; HNF: heterotrophic nanoflagellates; HDINO: heterotrophic dinoflagellates; CIL: ciliates. Abundances and biomass are given for particularly abundant taxa. Summer abundance and biomass data do not include surface slush samples; these data are reported in the text

\begin{tabular}{|c|c|c|c|c|}
\hline \multirow{2}{*}{ Group or taxon } & \multicolumn{2}{|c|}{ Abundance (cells $\mathrm{l}^{-1}$ ) } & \multicolumn{2}{|c|}{ Biomass $\left(\mu \mathrm{g} \mathrm{C} \mathrm{l}^{-1}\right.$ ) } \\
\hline & Autumn & Summer & Autumn & Summer \\
\hline $\begin{array}{l}\text { Autotrophic flagellates } \\
\text { (AFL) }\end{array}$ & $\begin{array}{c}2.1 \times 10^{5} \\
\left(3.9 \times 10^{3}-1.5 \times 10^{6}\right)\end{array}$ & $\begin{array}{c}8.8 \times 10^{5} \\
\left(2.6 \times 10^{4}-6.5 \times 10^{6}\right)\end{array}$ & $\begin{array}{c}3.5 \\
(0.1-23.1)\end{array}$ & $\begin{array}{c}24.6 \\
(0.4-256.2)\end{array}$ \\
\hline Pyramimonas spp. & $\left(1.2 \times 10^{2}-3.1 \times 10^{5}\right)$ & $\left(1.4 \times 10^{2}-5.8 \times 10^{6}\right)$ & $(0.0-7.0)$ & $(0.0-249.4)$ \\
\hline Cryptomonads & $\left(5.4 \times 10^{1}-1.1 \times 10^{5}\right)$ & $\left(1.5 \times 10^{1}-6.0 \times 10^{3}\right)$ & $(0.0-14.8)$ & $(0.0-1.0)$ \\
\hline Archaeomonads & $\left(1.2 \times 10^{2}-5.4 \times 10^{5}\right)$ & $\left(2.2 \times 10^{3}-1.1 \times 10^{6}\right)$ & $(1.0-1.0)$ & $(0.0-103.7)$ \\
\hline Mantoniella sp. & $\left(1.9 \times 10^{2}-1.3 \times 10^{6}\right)$ & $\left(1.2 \times 10^{3}-1.1 \times 10^{6}\right)$ & $(0.0-4.6)$ & $(0.1-8.0)$ \\
\hline Chrysophytes & $\left(6.7 \times 10^{1}-6.4 \times 10^{4}\right)$ & $\left(8.0 \times 10^{2}-9.9 \times 10^{5}\right)$ & $(0.0-0.6)$ & $(0.0-6.0)$ \\
\hline Parmales & $\left(8.9 \times 10^{2}-3.3 \times 10^{4}\right)$ & $\left(3.6 \times 10^{1}-2.2 \times 10^{5}\right)$ & $(0.0-0.8)$ & $(0.0-8.1)$ \\
\hline Miscellaneous autoflagellates & $\left(2.2 \times 10^{2}-1.8 \times 10^{5}\right)$ & $\left(1.3 \times 10^{4}-7.0 \times 10^{5}\right)$ & $(0.0-2.1)$ & $(0.1-7.5)$ \\
\hline $\begin{array}{l}\text { Phaeocystis (total) } \\
\text { (PHAEO) }\end{array}$ & $\begin{array}{c}2.6 \times 10^{5} \\
\left(2.1 \times 10^{3}-2.6 \times 10^{6}\right)\end{array}$ & $\begin{array}{c}2.3 \times 10^{5} \\
\left(2.7 \times 10^{3}-3.3 \times 10^{6}\right)\end{array}$ & $\begin{array}{c}1.3 \\
(0.1-9.6)\end{array}$ & $\begin{array}{c}9.4 \\
(0.2-84.0)\end{array}$ \\
\hline $\begin{array}{l}\text { Phaeocystis (non-motile) } \\
\text { Phaeocystis (motile) }\end{array}$ & $\left(2.0 \times 10^{3}-2.6 \times 10^{6}\right)$ & $\begin{array}{l}\left(1.1 \times 10^{3}-3.3 \times 10^{6}\right) \\
\left(1.4 \times 10^{3}-3.8 \times 10^{5}\right)\end{array}$ & $(0.1-9.6)$ & $\begin{array}{l}(0.2-84.0) \\
(0.1-4.2)\end{array}$ \\
\hline $\begin{array}{l}\text { Autotrophic dinoflagellates } \\
\text { (ADINO) }\end{array}$ & $\begin{array}{c}1.6 \times 10^{5} \\
\left(1.9 \times 10^{3}-2.1 \times 10^{6}\right)\end{array}$ & $\begin{array}{c}3.6 \times 10^{4} \\
\left(2.9 \times 10^{2}-4.9 \times 10^{5}\right)\end{array}$ & $\begin{array}{c}76.4 \\
(0.4-1349)\end{array}$ & $\begin{array}{c}16.4 \\
(0.8-85.2)\end{array}$ \\
\hline Amphidinium & $\left(4.1 \times 10^{3}\right)$ & & $(4.0)$ & \\
\hline Dinophysis & & $\left(3.0 \times 10^{4}\right)$ & & $(17.1)$ \\
\hline Gymnodinium & $\left(6.6 \times 10^{2}-1.7 \times 10^{6}\right)$ & $\left(1.8 \times 10^{2}-6.8 \times 10^{4}\right)$ & $(0.4-922.1)$ & $(0.2-70.2)$ \\
\hline Prorocentrum & $\left(6.4 \times 10^{1}-4.8 \times 10^{4}\right)$ & $\left(2.5 \times 10^{1}-9.9 \times 10^{4}\right)$ & $(0.8-6.4)$ & $(0.1-15.6)$ \\
\hline Cysts & $\left(5.0 \times 10^{0}-7.2 \times 10^{5}\right)$ & $\left(3.1 \times 10^{1}-2.1 \times 10^{5}\right)$ & $(0.0-19.6)$ & $(0.0-16.9)$ \\
\hline Miscellaneous thecate forms & $\left(6.4 \times 10^{1}-1.6 \times 10^{4}\right)$ & $\left(2.5 \times 10^{1}-1.2 \times 10^{5}\right)$ & $(0.0-31.5)$ & $(0.0-32.7)$ \\
\hline $\begin{array}{l}\text { Diatoms } \\
\text { (DIAT) }\end{array}$ & $\begin{array}{c}2.4 \times 10^{6} \\
\left(2.4 \times 10^{3}-3.0 \times 10^{7}\right)\end{array}$ & $\begin{array}{c}7.0 \times 10^{6} \\
\left(5.5 \times 10^{5}-3.5 \times 10^{7}\right)\end{array}$ & $\begin{array}{c}220.4 \\
(0.9-1612)\end{array}$ & $\begin{array}{c}441.8 \\
(9.6-4743)\end{array}$ \\
\hline Pseudo-nitzschia spp. & $\left(3.5 \times 10^{2}-1.9 \times 10^{4}\right)$ & $\left(1.3 \times 10^{5}-5.2 \times 10^{5}\right)$ & $(0.0-1.6)$ & $(0.7-184.4)$ \\
\hline P. turgiduloides & $\left(1.1 \times 10^{2}-9.5 \times 10^{5}\right.$ & $\left(3.5 \times 10^{1}-2.2 \times 10^{6}\right)$ & $(0.0-48.4)$ & $(0.0-137.5)$ \\
\hline P. subcurvata & $\left(2.4 \times 10^{2}-8.0 \times 10^{4}\right)$ & $\left(6.6 \times 10^{1}-7.1 \times 10^{5}\right)$ & $(0.0-2.1)$ & $(0.0-17.6)$ \\
\hline P. prolongitoides & & $\left(7.1 \times 10^{2}-5.1 \times 10^{4}\right)$ & & $(0.0-0.8)$ \\
\hline P. heimii & & $\left(4.8 \times 10^{1}-5.2 \times 10^{5}\right)$ & & $(0.0-183.4)$ \\
\hline$P$. lineola & & $\left(3.4 \times 10^{2}-6.3 \times 10^{4}\right)$ & & $(0.0-1.8)$ \\
\hline Nitzschia spp. & $\left(4.2 \times 10^{2}-2.6 \times 10^{7}\right)$ & & $(0.0-126.9)$ & \\
\hline N. lecointei & $\left(3.1 \times 10^{2}-1.5 \times 10^{6}\right)$ & $\left(3.6 \times 10^{2}-1.5 \times 10^{6}\right)$ & $(0.0-58.2)$ & $(0.0-55.1)$ \\
\hline N. longissima & $\left(1.5 \times 10^{2}-5.5 \times 10^{5}\right)$ & $\left(5.3 \times 10^{2}-7.9 \times 10^{5}\right)$ & $(0.0-12.1)$ & $(0.0-30.6)$ \\
\hline Navicula spp. & $\left(2.9 \times 10^{1}-7.8 \times 10^{4}\right)$ & $\left(6.7 \times 10^{1}-6.4 \times 10^{5}\right)$ & $(0.0-16.1)$ & $(0.0-113.1)$ \\
\hline Haslea sp. & $\left(6.3 \times 10^{1}-2.0 \times 10^{4}\right)$ & $\left(3.2 \times 10^{1}-2.0 \times 10^{2}\right)$ & $(0.0-9.0)$ & $(0.02-0.23)$ \\
\hline Fragilariopsis spp. & $\left(5.7 \times 10^{2}-9.4 \times 10^{5}\right)$ & $\left(1.3 \times 10^{3}-2.4 \times 10^{7}\right)$ & $(0.1-247.4)$ & $(0.0-2309.8)$ \\
\hline F. curta/cylindrus & $\left(3.1 \times 10^{2}-9.4 \times 10^{6}\right)$ & $\left(6.1 \times 10^{3}-2.4 \times 10^{7}\right)$ & $(0.0-731.5)$ & $(58.9-562.8)$ \\
\hline F. rhombica & $\left(7.3 \times 10^{1}-2.8 \times 10^{4}\right)$ & $\left(5.8 \times 10^{1}-2.2 \times 10^{4}\right)$ & $(0.0-5.0)$ & $(0.0-4.5)$ \\
\hline Entomoneis spp. & $\left(5.9 \times 10^{1}-1.1 \times 10^{4}\right)$ & $\left(3.2 \times 10^{1}-3.3 \times 10^{5}\right)$ & $(0.1-24.6)$ & $(0.1-1647.5)$ \\
\hline E. kjellmanii & $\left(5.2 \times 10^{1}-5.1 \times 10^{5}\right)$ & $\left(3.5 \times 10^{1}-2.2 \times 10^{5}\right)$ & $(0.1-1105.2)$ & $(0.1-439.4)$ \\
\hline Amphora spp. & $\left(2.8 \times 10^{5}-4.0 \times 10^{6}\right)$ & $\left(1.3 \times 10^{3}\right)$ & $(0.0-30.6)$ & $(0.1)$ \\
\hline Tropidoneis spp. & $\left(5.3 \times 10^{1}-6.4 \times 10^{3}\right)$ & $\left(3.0 \times 10^{1}-1.3 \times 10^{4}\right)$ & $(0.0-2.9)$ & $(0.0-6.0)$ \\
\hline Banquisia belgicae & $\left(7.5 \times 10^{1}-2.3 \times 10^{4}\right)$ & $\left(3.4 \times 10^{1}-2.8 \times 10^{4}\right)$ & $(0.0-30.6)$ & $(0.0-51.9)$ \\
\hline Membraneis challengeri & $\left(6.2 \times 10^{1}\right)$ & & $(0.1)$ & \\
\hline Manguinea rigida & & $\left(1.4 \times 10^{2}\right)$ & & $(0.1)$ \\
\hline Plagiotropis gaussii & $\left(3.3 \times 10^{2}-3.2 \times 10^{3}\right)$ & $\left(1.3 \times 10^{3}\right)$ & $(0.4-2.7)$ & $(0.9)$ \\
\hline Manuinea fusiformis & $\left(2.1 \times 10^{2}-3.4 \times 10^{5}\right)$ & $\left(6.4 \times 10^{1}-7.0 \times 10^{5}\right)$ & $(0.0-80.4)$ & $(0.0-209.8)$ \\
\hline Synedripsis hyperborea & $\left(6.3 \times 10^{1}-1.2 \times 10^{5}\right)$ & $\left(2.5 \times 10^{0}-2.6 \times 10^{4}\right)$ & $(0.0-5.8)$ & $(0.0-5.5)$ \\
\hline Trichotoxon reinboldii & $\left(2.9 \times 10^{1}-1.5 \times 10^{5}\right)$ & $\left(5.5 \times 10^{1}\right)$ & $(0.0-355.5)$ & $(0.0)$ \\
\hline Pleurosigma sp. & & $\left(4.3 \times 10^{1}-9.9 \times 10^{4}\right)$ & & $(0.1-218.8)$ \\
\hline Miscellaneous Pennates & $\left(4.3 \times 10^{1}-6.6 \times 10^{4}\right)$ & $\left(1.1 \times 10^{2}-7.6 \times 10^{5}\right)$ & $(0.0-25.2)$ & $(0.0-288.1)$ \\
\hline Eucampia antarctica & $\left(3.7 \times 10^{1}-2.3 \times 10^{3}\right)$ & $\left(6.4 \times 10^{1}-3.7 \times 10^{4}\right)$ & $(0.1-3.1)$ & $(0.0-62.2)$ \\
\hline Odontella weissflogii & $\left(4.8 \times 10^{1}-6.2 \times 10^{1}\right)$ & $\left(9.7 \times 10^{1}-8.4 \times 10^{3}\right)$ & $(0.1-0.1)$ & $(0.2-26.0)$ \\
\hline Chaetoceros spp. & $\left(2.9 \times 10^{1}-6.7 \times 10^{4}\right)$ & $\left(5.4 \times 10^{1}-1.5 \times 10^{3}\right)$ & $(0.0-10.1)$ & $(0.0-0.0)$ \\
\hline
\end{tabular}


Table 1 (continued)

\begin{tabular}{|c|c|c|c|c|}
\hline \multirow[t]{2}{*}{ Group or taxon } & \multicolumn{2}{|c|}{ Abundance (cells ${ }^{-1}$ ) } & \multicolumn{2}{|c|}{ Biomass $\left(\mu g \mathrm{C}^{-1}\right)$} \\
\hline & Autumn & Summer & Autumn & Summer \\
\hline Chaetoceros alanticum & $\left(1.6 \times 10^{2}-7.9 \times 10^{4}\right)$ & $\left(1.1 \times 10^{2}\right)$ & $(0.0-19.5)$ & $(0.1)$ \\
\hline C. bulbosum & $\left(3.2 \times 10^{1}-3.3 \times 10^{3}\right)$ & $\left(1.3 \times 10^{2}-4.4 \times 10^{2}\right)$ & $(0.0-30.9)$ & $(0.0-0.0)$ \\
\hline C. criophilum & & $\left(1.1 \times 10^{2}-8.8 \times 10^{2}\right)$ & & $(0.1-0.4)$ \\
\hline C. flexuosum & $\left(6.7 \times 10^{2}-1.4 \times 10^{4}\right)$ & & $(0.1-3.2)$ & \\
\hline C. dichaeta & $\left(1.2 \times 10^{1}-5.2 \times 10^{5}\right)$ & $\left(1.4 \times 10^{2}-5.2 \times 10^{5}\right)$ & $(0.0-70.0)$ & $(0.0-64.0)$ \\
\hline C. neglectum & $\left(2.9 \times 10^{1}-3.0 \times 10^{5}\right)$ & $\left(3.6 \times 10^{2}-1.7 \times 10^{3}\right)$ & $(0.0-14.6)$ & $(0.0-0.0)$ \\
\hline C. neogracile & $\left(4.0 \times 10^{3}-6.9 \times 10^{4}\right)$ & $\left(2.9 \times 10^{4}-8.8 \times 10^{6}\right)$ & $(0.5-1.9)$ & $(0.7-215.9)$ \\
\hline C. socialis & & $\left(1.3 \times 10^{2}-2.1 \times 10^{4}\right)$ & & $(0.0-1.3)$ \\
\hline Actinocyclus sp. & $\left(3.1 \times 10^{1}-1.6 \times 10^{2}\right)$ & $\left(2.9 \times 10^{2}-5.8 \times 10^{5}\right)$ & $(0.0-0.2)$ & $(0.3-334.7)$ \\
\hline Coscinodiscus sp. & $\left(3.1 \times 10^{1}-5.2 \times 10^{3}\right)$ & $\left(8.0 \times 10^{1}-7.5 \times 10^{3}\right)$ & $(0.1-7.2)$ & $(0.6-74.9)$ \\
\hline Thalassiosira sp. & $\left(8.4 \times 10^{1}-3.3 \times 10^{2}\right)$ & $\left(2.0 \times 10^{2}-2.5 \times 10^{5}\right)$ & $(0.1-1.1)$ & $(0.6-420.5)$ \\
\hline Rhizosolenia spp. & $\left(4.0 \times 10^{1}-5.4 \times 10^{5}\right.$ & $\left(3.2 \times 10^{1}-2.0 \times 10^{5}\right.$ & $(0.0-253.3)$ & $(0.0-624.5)$ \\
\hline$R$. bergonii & & $\left(1.1 \times 10^{3}-1.1 \times 10^{4}\right)$ & & $(4.4-61.1)$ \\
\hline Proboscia alata & & $\left(1.8 \times 10^{2}\right)$ & & $(0.1)$ \\
\hline P. truncata & & $\left(7.7 \times 10^{1}-1.8 \times 10^{4}\right)$ & & $(0.1-48.0)$ \\
\hline Dactyliosolen antarcticus & $\left(3.7 \times 10^{1}-5.2 \times 10^{4}\right)$ & $\left(4.3 \times 10^{1}-5.0 \times 10^{4}\right)$ & $(0.0-76.2)$ & $(0.1-16.3)$ \\
\hline D. tenuijuctus & $\left(3.1 \times 10^{1}-1.9 \times 10^{3}\right)$ & $\left(6.8 \times 10^{1}-1.2 \times 10^{4}\right)$ & $(0.0-0.4)$ & $(0.0-3.5)$ \\
\hline Corethron criophilum & $\left(1.1 \times 10^{2}-6.6 \times 10^{5}\right)$ & $\left(3.3 \times 10^{1}-5.3 \times 10^{4}\right)$ & $(0.0-477.5)$ & $(0.0-62.2)$ \\
\hline Miscellaneous centrics & $\left(2.0 \times 10^{0}-3.6 \times 10^{3}\right)$ & $\left(4.8 \times 10^{1}-1.2 \times 10^{5}\right)$ & $(0.1-3.7)$ & $(0.0-275.4)$ \\
\hline $\begin{array}{l}\text { Heterotrophic flagellates } \\
\text { (HNF) }\end{array}$ & $\begin{array}{c}6.6 \times 10^{5} \\
\left(3.9 \times 10^{3}-1.2 \times 10^{7}\right)\end{array}$ & $\begin{array}{c}1.8 \times 10^{6} \\
\left(1.1 \times 10^{5}-1.0 \times 10^{7}\right)\end{array}$ & $\begin{array}{l}22.1 \\
(0.0-588.2)\end{array}$ & $\begin{array}{c}31.5 \\
(1.2-113.5)\end{array}$ \\
\hline Choanoflagellates & $\left(7.5 \times 10^{2}-1.3 \times 10^{6}\right.$ & $\left(2.1 \times 10^{3}-2.1 \times 10^{6}\right)$ & $(0.0-7.1)$ & $(0.0-30.2)$ \\
\hline Teleonema antarctica & $\left(8.2 \times 10^{2}-3.3 \times 10^{5}\right)$ & $\left(2.9 \times 10^{2}-2.9 \times 10^{5}\right)$ & $(0.0-1.7)$ & $(0.0-2.2)$ \\
\hline T. subtilis & $\left(4.2 \times 10^{2}-1.8 \times 10^{5}\right)$ & $\left(6.9 \times 10^{2}-1.7 \times 10^{5}\right)$ & $(0.0-2.5)$ & $(0.0-9.1)$ \\
\hline Cryothecomonas & $\left(5.0 \times 10^{0}-4.1 \times 10^{6}\right)$ & $\left(4.4 \times 10^{1}-1.2 \times 10^{5}\right)$ & $(0.0-440.0)$ & $(0.0-18.5)$ \\
\hline Bicosoeca spp. & & $\left(1.4 \times 10^{3}-3.8 \times 10^{5}\right)$ & & $(0.0-5.0)$ \\
\hline Euglenoid & $\left(1.1 \times 10^{1}-1.0 \times 10^{6}\right)$ & $\left(1.6 \times 10^{3}-8.3 \times 10^{5}\right)$ & $(0.01-29.4)$ & $(0.1-67.9)$ \\
\hline Anisonema sp. & $\left(5.3 \times 10^{1}-4.3 \times 10^{4}\right)$ & $\left(1.4 \times 10^{1}-1.6 \times 10^{4}\right)$ & $(0.1-7.4)$ & $(0.0-8.4)$ \\
\hline Cryptaulax sp. & $\left(2.2 \times 10^{2}-3.5 \times 10^{4}\right)$ & $\left(5.1 \times 10^{3}-3.5 \times 10^{4}\right)$ & $(0.0-0.8)$ & $(0.1-0.7)$ \\
\hline Amastigomonas sp. & & $\left(2.1 \times 10^{3}-3.0 \times 10^{5}\right)$ & & $(0.1-9.5)$ \\
\hline Paraphysomonas sp. & $\left(1.6 \times 10^{2}-4.2 \times 10^{2}\right)$ & & $(0.0-0.0)$ & \\
\hline Leucocryptos sp. & $\left(2.6 \times 10^{2}-1.0 \times 10^{4}\right)$ & & $(0.0-0.2)$ & \\
\hline Bodo sp. & $\left(4.1 \times 10^{2}-3.2 \times 10^{4}\right)$ & & $(0.0-0.1)$ & \\
\hline Petalomonas sp. & $\left(6.7 \times 10^{1}-2.0 \times 10^{3}\right)$ & & $(0.0-0.1)$ & \\
\hline Quadricilia sp. & $\left(9.0 \times 10^{3}-3.0 \times 10^{4}\right)$ & & $(0.1-0.3)$ & \\
\hline Miscellaneous flagellates & $\left(8.2 \times 10^{2}-5.6 \times 10^{6}\right)$ & $\left(8.1 \times 10^{4}-8.0 \times 10^{6}\right)$ & $(0.0-110.5)$ & $(0.4-93.6)$ \\
\hline $\begin{array}{l}\text { Heterotrophic dinoflagellates } \\
\text { (HDINO) }\end{array}$ & $\begin{array}{c}2.9 \times 10^{4} \\
\left(4.4 \times 10^{1}-2.7 \times 10^{5}\right)\end{array}$ & $\begin{array}{c}1.2 \times 10^{4} \\
\left(6.1 \times 10^{2}-2.0 \times 10^{5}\right)\end{array}$ & $\begin{array}{l}12.0 \\
(0.0-140.3)\end{array}$ & $\begin{array}{c}3.3 \\
(0.1-28.0)\end{array}$ \\
\hline Gymnodinium spp. & $\left(3.3 \times 10^{1}-1.8 \times 10^{5}\right)$ & $\left(4.1 \times 10^{2}-3.2 \times 10^{4}\right)$ & $(0.0-122.0)$ & $(0.0-28.0)$ \\
\hline Katodinium spp. & $\left(2.2 \times 10^{1}-8.5 \times 10^{4}\right)$ & $\left(1.3 \times 10^{1}-1.9 \times 10^{5}\right)$ & $(0.0-57.1)$ & $(0.0-3.1)$ \\
\hline Amphidinium spp. & $\left(4.2 \times 10^{2}\right)$ & $\left(4.7 \times 10^{1}\right)$ & $(0.4)$ & $(0.01)$ \\
\hline Prorocentrum spp. & $\left(1.5 \times 10^{2}-8.2 \times 10^{3}\right)$ & & $(0.0-1.3)$ & \\
\hline Protoperidinium spp. & $\left(2.9 \times 10^{1}-5.0 \times 10^{2}\right)$ & & $(0.0-4.0)$ & \\
\hline Miscellaneous dinoflagellates & $\left(4.4 \times 10^{1}-2.7 \times 10^{5}\right)$ & $\left(6.1 \times 10^{2}-2.0 \times 10^{5}\right)$ & $(0.0-140.3)$ & $(0.1-27.9)$ \\
\hline $\begin{array}{l}\text { Ciliates } \\
\text { (CIL) }\end{array}$ & $\begin{array}{c}5.2 \times 10^{3} \\
\left(9.5 \times 10^{1}-4.0 \times 10^{4}\right)\end{array}$ & $\begin{array}{c}2.0 \times 10^{3} \\
\left(1.7 \times 10^{2}-7.3 \times 10^{3}\right)\end{array}$ & $\begin{array}{l}29.2 \\
(0.0-141.4)\end{array}$ & $\begin{array}{c}37.2 \\
(0.0-309.7)\end{array}$ \\
\hline Strombidium & $\left(2.0 \times 10^{1}-2.5 \times 10^{3}\right)$ & $\left(3.4 \times 10^{1}-8.2 \times 10^{2}\right)$ & $(0.0-9.4)$ & $(0.0-6.6)$ \\
\hline Spirostrombidium & & $\left(4.5 \times 10^{1}-6.8 \times 10^{1}\right)$ & & $(0.1-2.8)$ \\
\hline Myrionecta & & $\left(2.8 \times 10^{1}-4.0 \times 10^{2}\right)$ & & $(0.0-0.8)$ \\
\hline Cytharoides & & $\left(4.8 \times 10^{1}-6.6 \times 10^{1}\right)$ & & $(11.8-39.0)$ \\
\hline Euplotes & $\left(3.1 \times 10^{1}-3.6 \times 10^{2}\right)$ & $\left(3.3 \times 10^{1}-2.2 \times 10^{3}\right)$ & $(0.0-14.1)$ & $(0.0-31.1)$ \\
\hline Lacrymaria & $\left(3.1 \times 10^{1}-2.6 \times 10^{3}\right)$ & $\left(3.6 \times 10^{1}-7.5 \times 10^{2}\right)$ & $(0.0-6.2)$ & $(0.3-42.0)$ \\
\hline Litonotus & $\left(5.2 \times 10^{1}-1.1 \times 10^{2}\right)$ & & $(0.8-2.5)$ & \\
\hline Tintinnid & $\left(1.5 \times 10^{2}-9.3 \times 10^{2}\right)$ & & $(0.3-7.0)$ & \\
\hline Gymnozoum & $\left(7.0 \times 10^{1}-4.8 \times 10^{3}\right)$ & $\left(3.2 \times 10^{1}-1.3 \times 10^{3}\right)$ & $(0.6-34.1)$ & $(0.1-23.5)$ \\
\hline Didinium & & $\left(4.3 \times 10^{1}-5.8 \times 10^{1}\right)$ & & $(0.7-6.0)$ \\
\hline Uronema & & $\left(3.3 \times 10^{1}-5.9 \times 10^{2}\right.$ & & $(0.0-0.7)$ \\
\hline Hypotrich & $\left(2.4 \times 10^{2}-5.6 \times 10^{2}\right)$ & & $(0.2-47.3)$ & \\
\hline Pleurotrich & $\left(6.5 \times 10^{1}-2.8 \times 10^{2}\right)$ & & $(0.3-10.4)$ & \\
\hline Miscellaneous ciliates & $\left(6.7 \times 10^{1}-3.8 \times 10^{4}\right)$ & $\left(1.4 \times 10^{2}-6.5 \times 10^{3}\right)$ & $(0.0-119.5)$ & $(0.8-272.4)$ \\
\hline
\end{tabular}


A minimum of 30 to 50 cells of each taxon were counted from random transects except when cells were rare. To estimate cell carbon content, we chose appropriate geometric shapes to represent cells and measured the critical dimensions required to calculate cell volumes. To convert diatom and flagellate cell volume to carbon biomass, we used the modified Strathmann equations (Eppley et al. 1970), where diatom carbon $(\mathrm{pg})=\log _{10}\left[0.94 \times \log _{10}(\right.$ volume in $\left.\mu^{3}\right)$ - 0.6] and flagellate carbon $(\mathrm{pg})=\log _{10}[0.76 \times$ $\log _{10}$ (volume in $\mu^{3}$ ) -0.352$]$. When Phaeocystis colonies were present, we measured the volume of colonies and used the colony volume relationship (colony volume $\left[\mu^{3}\right]=417 \times$ cell number ${ }^{0.6}$ ) to determine the cell per colony density (Mathot et al. 2000). For both colony and free, motile cells of Phaeocystis, we used the modified Strathman flagellate relationship cited above to determine carbon cell ${ }^{-1}$. For estimating ciliate biomass, we used the relationship pg carbon $=0.16 \times\left(\right.$ cell volume in $\left.\mu^{3}\right)$ and pg carbon $=$ $0.19 \times\left(\right.$ cell volume in $\mu^{3}$ ) for samples preserved with paraformaldehyde and Lugol's iodine, respectively (Putt \& Stoecker 1989). Organisms were identified to the lowest taxon we were able to assign during routine counting (see Table 1). Many of the taxa listed in Table 1 have been described over more than a decade of studies of the Antarctic sea-ice biota (e.g. Corliss \& Snyder 1986, Marchant et al. 1989, Buck et al. 1990a,b, 1992, Thomsen et al. 1990, 1991, 1997 , Daugbjerg et al. 2000), and many can be recognized with reasonable certainty during counts using either light or epifluorescence microscopy. When necessary, identifications were confirmed (usually using special sample preparations) by light and scanning electron microscopy as appropriate.

Community analyses procedures, similar to those outlined by Field et al. (1982), were used to examine the similarity of assemblage composition between stations and cruises, and to assess which taxa were responsible for the variations. Biomass was classified into 7 major taxonomic groups as shown in Table 1. Similarities were calculated between paired stations as the Percent Similarity Index (PSI):

$\operatorname{PSI}\left(\operatorname{Sample}_{\mathrm{a}, \mathrm{b}}\right)=\sum$ Minimum $\left[\operatorname{Group}_{i}\left(\% \operatorname{Sample}_{\mathrm{a}}{ } \%\right.\right.$ Sample $\left.\left._{\mathrm{b}}\right)\right]$

where $i=($ groups $1,2 \ldots .7)$ in a comparison of the similarity between samples a and b (Washington 1984). The resulting station-by-station similarity matrix was initially examined by using a single-linkage clustering routine and then using multi-dimensional scaling (MDS) (Field et al. 1982). After identifying distinct station groups, the individual group contributions to each paired-station comparison were normalized by the PSI value for the comparison, and the average within-cluster contribution to the overall similarity was assessed for each station-cluster. These analyses allow an objective identification of the taxa that are responsible for the clustering patterns among stations.

Integrated biomass (derived from microcopy) was calculated for NBP98-3 samples from sequential sections throughout ice floes. For NBP99-1, we collected and analyzed samples only at selected intervals from the surface, interior, and bottom of cores for microscopy analysis (usually 3 to 4 sections per core). To obtain estimates of integrated microscopicallyderived biomass over the thickness of the ice, values were either extrapolated from the values of the adjacent core sections, or we averaged the values between segments where we had data. An examination of the complete chl a profiles was used to determine whether an extrapolation or an averaging procedure was most appropriate. The rationale for using chl a profiles as a guide to either extrapolate or average the data on autotrophs is clear. We have also consistently found strong positive correlations between autotrophic and heterotrophic biomass in sea ice from a number of locations and during different seasons (data not shown), suggesting that, lacking other criteria, it is justified to use chl a profiles to guide the calculation of integrated heterotrophic biomass.

\section{RESULTS}

\section{Autumn (NBP98-3)}

The autumn cruise (NBP98-3) took place midway in the transition from autumn to winter conditions (Table 2). During this period, the ice cover was increasing in area and thickness, daylight and air temperatures were decreasing, resulting in lower temperatures within ice, higher brine salinities, and lower brine volumes, the most extreme values commonly occurring in the upper part of the ice floes.

Integrated autotrophic biomass (nanoflagellates, Phaeocystis, dinoflagellates, and diatoms) in floes 40 to $90 \mathrm{~cm}$ in thickness ranged from 1.6 to $708.6 \mathrm{mg} \mathrm{C} \mathrm{m}^{-2}$. Integrated heterotrophic biomass (flagellates, heterotropic dinoflagellates, and ciliates) ranged from 0.7 to $166.7 \mathrm{mg} \mathrm{C} \mathrm{m}^{-2}$. Autotrophic biomass dominated most assemblages with a median of 82 and a range of 48 to $96 \%$ of the biomass (Table 2). Abundance and biomass for discrete-depth intervals showed considerable variability (Table 1). C:chl a ratios averaged 33 (median = 28) and ranged from 2 to 114

Chl a concentrations showed marked spatial variation with most well-developed algal concentrations in floes in the northern part of the study area (Fig. 2). Chl a within floes ranged from 0.2 to $20.9 \mathrm{mg} \mathrm{m}^{-2}$ (Table 2, Fig. 2). The overall pattern of microscopi- 
Table 2. Summary of environmental conditions, the chemical and physical characteristics, and the biomass parameters measured within sea-ice floes. Carbon values cited are from microscopy estimates. Since day length is a function of both season and latitude, the minimum and maximum for each of the cruises are shown. Values for the surface slush assemblages that were observed only during the summer cruise are not included, but these values are discussed in the text

\begin{tabular}{|c|c|c|c|}
\hline Parameter & Min. & Median & Max. \\
\hline \multicolumn{4}{|c|}{ Autumn to winter transition (NBP98-3) } \\
\hline \multicolumn{4}{|l|}{ Environmental parameters } \\
\hline Day length (h) & 0 & - & 6 \\
\hline Air temperature $\left({ }^{\circ} \mathrm{C}\right)$ & -33 & - & +0.5 \\
\hline \multicolumn{4}{|l|}{ Sea ice characteristics } \\
\hline Ice thickness (cm) & 28 & 69 & 117 \\
\hline Snow depth $(\mathrm{cm})$ & 0 & 10 & 36 \\
\hline Freeboard $(\mathrm{cm})$ & -4 & 2 & 11 \\
\hline Brine salinity (psu) & 32 & 85 & 180 \\
\hline Brine volume $(\%)$ & 3 & 8 & 21 \\
\hline \multicolumn{4}{|l|}{ Biomass parameters } \\
\hline Chl a $\left(\mu g \mathrm{l}^{-1}\right)$ in ice & 0.0 & 0.6 & 96.9 \\
\hline Chl a $\left(\mathrm{mg} \mathrm{m}^{-2}\right)$ in ice & 0.2 & 0.4 & 20.9 \\
\hline $\begin{array}{l}\text { Autotrophic biomass } \\
\left(\mathrm{mg} \mathrm{C} \mathrm{m}^{-2}\right)\end{array}$ & 1.6 & 86.9 & 708.6 \\
\hline Heterotrophic biomass & & & \\
\hline$\left(\mathrm{mg} \mathrm{C} \mathrm{m}^{-2}\right)$ & 0.7 & 10.1 & 166.7 \\
\hline Autotrophs/total biomass & 0.48 & 0.82 & 0.96 \\
\hline Carbon (autotrophs)/chl a & 2 & 28 & 114 \\
\hline \multicolumn{4}{|l|}{ Summer (NBP99-1) } \\
\hline \multicolumn{4}{|l|}{ Environmental parameters } \\
\hline Day length (h) & 17 & - & 24 \\
\hline Air temperature $\left({ }^{\circ} \mathrm{C}\right)$ & -6.3 & - & +3.7 \\
\hline \multicolumn{4}{|l|}{ Sea-ice characteristics } \\
\hline Ice thickness (cm) & 40 & 127 & 260 \\
\hline Snow depth (cm) & 3.5 & 27 & 51 \\
\hline Freeboard (cm) & -26 & 0 & 22 \\
\hline Brine salinity (psu) & 1.8 & 28.7 & 39.1 \\
\hline Brine volume $(\%)$ & 7.5 & 15.0 & 34 \\
\hline \multicolumn{4}{|l|}{ Biomass parameters } \\
\hline $\mathrm{Chl} \mathrm{a}\left(\mu \mathrm{g} \mathrm{l^{-1 }}\right)$ & 0.1 & 5.2 & 84.5 \\
\hline $\mathrm{Chl} a\left(\mathrm{mg} \mathrm{m}^{-2}\right)$ & 2.0 & 6.0 & 40.7 \\
\hline $\begin{array}{l}\text { Autotrophic biomass } \\
\left(\mathrm{mg} \mathrm{C} \mathrm{m}^{-2}\right)\end{array}$ & 102.3 & 259.3 & 3873 \\
\hline $\begin{array}{l}\text { Heterotrophic biomass } \\
\left(\mathrm{mg} \mathrm{C} \mathrm{m}^{-2}\right)\end{array}$ & 9.2 & 65.3 & 209.3 \\
\hline Autotrophs/total biomass & 0.57 & 0.84 & 0.98 \\
\hline Carbon (autotrophs)/chl a & 11 & 55 & 223 \\
\hline
\end{tabular}

cally-derived biomass (Fig. 3) generally followed the same pattern shown by chl a (Fig. 2). Diatoms most often dominated the autotrophic biomass (average $=$ $74 \%$; range $=29$ to $97 \%$ ), followed by dinoflagellates (average $=20 \%$; range $=1$ to $64 \%$ ) and autotrophic flagellates (average $=4 \%$; range $=<1$ to $15 \%$ ). Phaeocystis made up about $1 \%$ of the autotrophic biomass (range $=<1$ to $3 \%$ ). Among the heterotrophic organisms, ciliates made up an average of $71 \%$, (range $=36$ to $87 \%$ ) followed by heterotrophic flagellates (aver- age $=16 \%$; range $=5$ to $44 \%$ ) and dinoflagellates (average $=13 \%$; range $=0$ to $30 \%$ ).

Clustering of stations based on similarity of biomass composition, expressed as PSI, indicated a high degree of similarity, with the PSI among all combinations of stations (45 combinations of PSI analysis) averaging $63 \%$. Single-linkage clustering analysis (dendrogram not shown) showed all stations entering clusters at PSI $>70 \%$ and 2 additional clusters forming at $>80 \%$ similarity. The relationship among clusters is shown in the MDS plot of the similarity matrix (Fig. 4). Because the PSI analysis is most heavily weighted by the biomass dominant, and overall diatoms dominated sea-ice microbial communities, diatom variability accounted for most of the clustering relationships (Table 3). An examination of the similarity calculations suggested that diatoms, then ciliates, and then autotrophic dinoflagellates accounted for the clustering patterns. This analysis also suggested that Cluster A was differentiated from Cluster B by having a higher proportion of diatoms, and a lesser proportion of ciliates and autotrophic dinoflagellates (see Table 3). Stn 160, most similar to Group B (Fig. 4), was distinguished from that group by having a higher proportion of autotrophic dinoflagellates (see Fig. 3).

At least 20 diatom species were abundant and/or common (Table 1), including Entomoneis spp. and Fragiliariopis (curta and/or cylindrus), which are typical for ice assemblages, as well as usually 'planktonic' forms such as Rhizosolenia spp., Dactyliosolen, Corethron, Trichotoxon reinboldii, and Chaetoceros dichaeta. Moderate abundances of Gymnodinium spp. occurred in several samples, particularly those from the southern part of the transect, and dinoflagellate

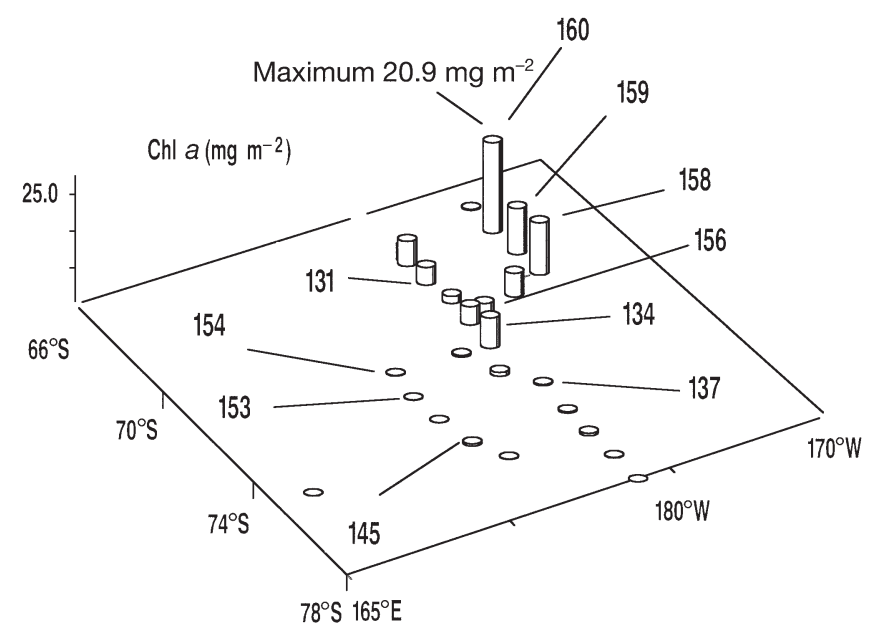

Fig. 2. Integrated $\mathrm{chl} \mathrm{a}\left(\mathrm{mg} \mathrm{m}^{-2}\right)$ in floes during NBP98-3. Stations analyzed for community structure are identified. See Fig. 1 for a more accurate station location 


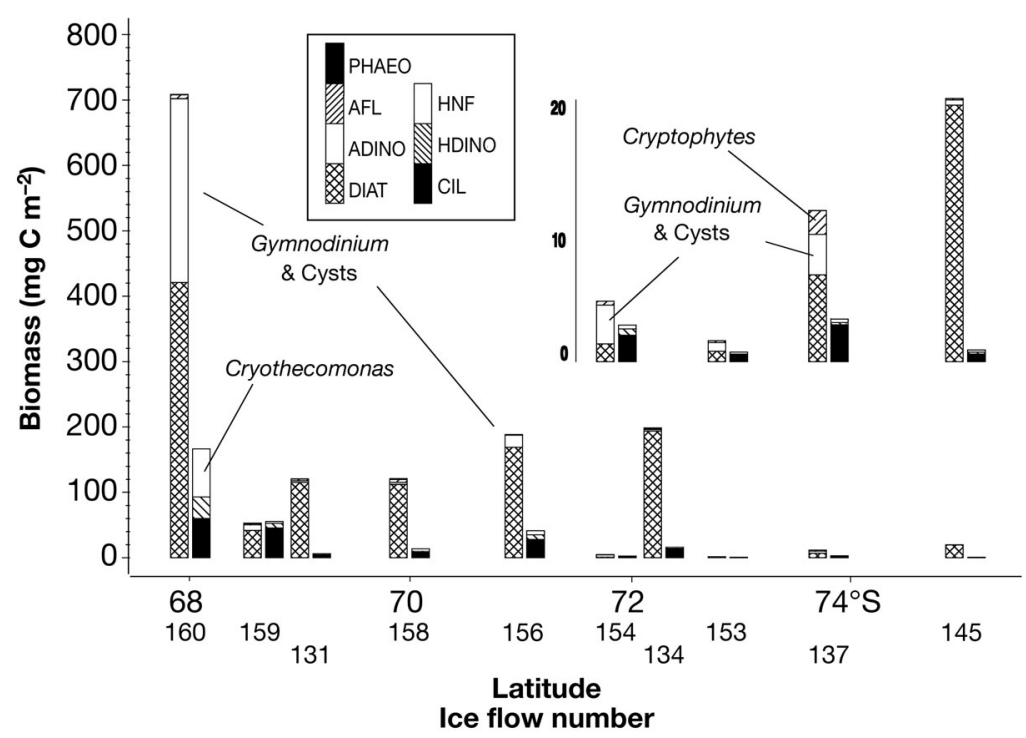

Fig. 3. Integrated biomass in floes sampled during NBP98-3. The first bar is autotrophic biomass; the second bar is heterotrophic biomass. Note that several stations with biomass too low to resolve groups are shown with the scale expanded 20x above the full-scale plots. Prominent species are identified for some stations. See 'Results, Autumn (NBP98-3)' and Table 1 for abbreviations

resting cysts also occurred in many of these samples. Stn 137 was somewhat unusual because Cryptomonads were abundant.

\section{Summer (NBP99-1)}

Cruise NBP99-1 was conducted to the east of the NBP98-3 study area (see Fig. 1), primarily because the latter included the Ross Sea Polynya region, and by summer this was largely ice-free. In contrast to the winter cruise, multi-year ice was found and sampled (Stns 008, 017, and 028; see Fig. 1) during NBP99-1. This cruise began about $8 \mathrm{~d}$ after the austral summer solstice. This period was characterized by long days and higher air temperatures (Table 2), resulting in higher ice temperatures, with corresponding lower brine salinities and higher brine volumes, relative to winter conditions. Descriptions of the ice communities observed during this cruise have been presented in Fritsen et al. (2001).

Integrated autotrophic biomass in ice cores from 40 to $260 \mathrm{~cm}$ in thickness ranged from 102.3 to $3873 \mathrm{mg} \mathrm{C}$ $\mathrm{m}^{-2}$. Heterotrophic biomass ranged from 9.2 to 209.3 $\mathrm{mg} \mathrm{C} \mathrm{m}{ }^{-2}$. Autotrophs consistently dominated the assemblages $($ median $=84 \%$; range $=57$ to $98 \%$ ). As was also noted for the winter samples, the abundances and biomass for discrete-depth intervals in core samples showed considerable variability (Table 1). C:chl a ratios (from discrete-depth samples) ranged from 11 to
223 , with a mean value of $43($ median $=55)$ (Table 2).

Depth-integrated chl a within floes ranged from 2.0 to $40.7 \mathrm{mg} \mathrm{m}^{-2}$ (Table 2, Fig. 5). Diatoms consistently dominated the autotrophic component of the sea-ice biota (average $=86 \%$; range $=64$ to $97 \%)$, followed by autotrophic dinoflagellates (average $=6 \%$; range $=<1$ to $31 \%$ ), autotrophic flagellates (average $=6 \%$; range $=<1$ to $31 \%$ ), and Phaeocystis (average $=3 \%$; range $=<1$ to $6 \%$ ). Among heterotrophs, ciliates predominated in most samples (average $=51 \%$; range $=13$ to $72 \%)$, followed by heterotrophic flagellates (average $=42 \%$; range $=21$ to $76 \%$ ) and heterotrophic dinoflagellates (average $=7 \%$; range $=<2$ to $26 \%$ ).

In contrast to the autumn cruise, where most of the apparent biomass was within the solid ice floes, during the summer cruise (NBP99-1) blooms of surface-layer slush populations were apparent throughout the study region (Fritsen et al. 2001). As was the case with populations within the ice, surface layer slush was dominated by autotrophic forms (average $=74 \%$; range $=18$ to 98 ). Although these assemblages were usually diatom-dominated (average $=$

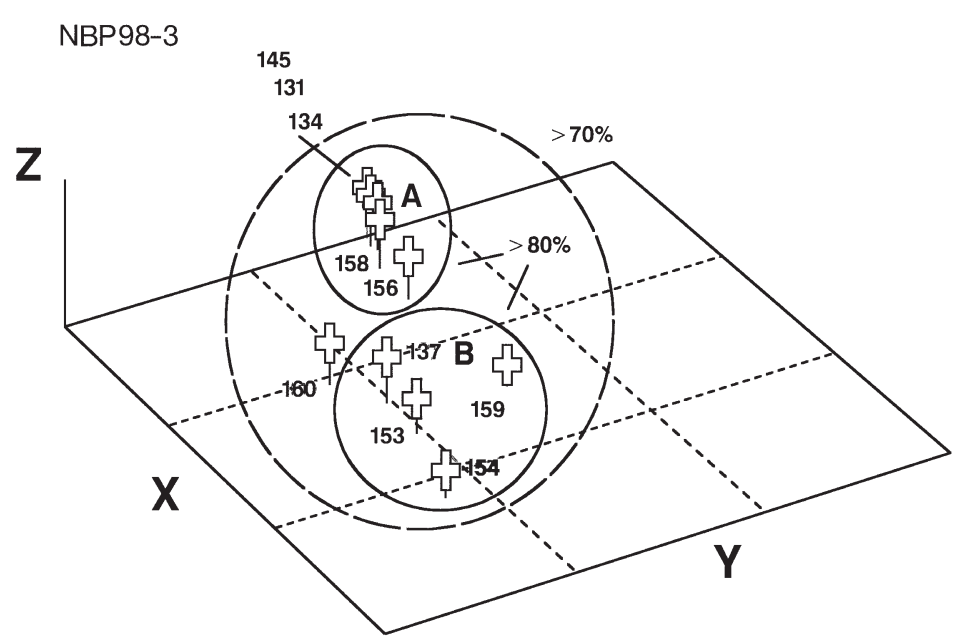

Fig. 4. Multidimensional scaling plots (MDS) of percent similarity matrix (PSI) based on the composition of the biomass. The large dashed circle indicates stations that clustered at the $70 \%$ similarity level. Solid lines show 2 station-groups (A and B) that clustered at the $80 \%$ similarity level. Station 160 was not included in either group at the $80 \%$ level. Values for axes $\mathrm{x}, \mathrm{y}$, and $\mathrm{z}$ are relative distance and are not shown. However, the comparative plot for NBP99-1 (Fig. 7) has been plotted with the same range of values, so these can be directly compared 
Table 3. Contribution of major groups to cluster similarity for autumn samples. The individual contribution of major groups to the percent similarity analysis (PSI). For each comparison within the similarity matrix, the group contribution to the PSI was normalized by the total PSI for the sample (see 'Materials and methods'). The comparisons shown are among all core samples (ice floes) and within clusters (A and B) identified in

Fig. 4. See Table 1 for abbreviations

\begin{tabular}{|lccc|}
\hline & $\begin{array}{c}\text { Ice floes } \\
(45)\end{array}$ & $\begin{array}{c}\text { Cluster A } \\
(10)\end{array}$ & $\begin{array}{c}\text { Cluster B } \\
(6)\end{array}$ \\
\hline & & & \\
DIAT & 0.64 & 0.75 & 0.64 \\
CIL & 0.15 & 0.12 & 0.20 \\
HNF & 0.05 & 0.01 & 0.03 \\
ADINO & 0.09 & 0.06 & 0.10 \\
AFL & 0.03 & 0.03 & 0.03 \\
PHAEO & 0.01 & 0.01 & 0.01 \\
HDINO & 0.02 & 0.01 & 0.01 \\
& & & \\
\hline
\end{tabular}

$44 \%$; range $=2$ to $95 \%$ ), autotrophic dinoflagellates (average $=14 \%$; range $=<1$ to $95 \%$ ), Phaeocystis (average $=30$; range $=0$ to $75 \%$ ), and autotrophic flagellates (average $=12 \%$; range $=<1$ to $97 \%$ ) were relatively more important than in the underlying ice and sometimes predominated (discussed in more detail below).

Chl a concentrations in the surface slush assemblage ranged from 0.08 to $60.5 \mu \mathrm{g} \mathrm{l}^{-1}$. At most stations, the chlorophyll-estimated biomass within the underlying floes exceeded that of the overlying slush (slush averaged $21 \%$ of the integrated stocks in cores). However, at 2 stations (Stns 010 and 011), slush autotrophic biomass (estimated as chl a) exceeded that contained in the underlying ice. At 4 stations, where slush versus ice comparisons based on microscopy could be made (Stns 004, 005, 009, and 017), total biomass in slush, as a fraction of the total biomass in ice plus slush, was 19, 26, 19, and $12 \%$, respectively. At these stations, the biomass of autotrophic flagellates, Phaeocystis, autotrophic dinoflagellates, and less frequently heterotrophic flagellates or heterotrophic dinoflagellates, in the slush layers sometimes exceeded the stocks within the underlying ice.

The integrated biomass of the ice microbial assemblages varied over the study region (Fig. 6A-C). The highest values were observed in stations on the southerly transect along both $150^{\circ} \mathrm{W}$ (Fig. 6B) and $135^{\circ} \mathrm{W}$ (Fig. 6C). Stn 030 was unusual in that the biomass exceeded that of other stations by at least 8 -fold. Multi-year floe 017 showed no apparent differences from younger floes with respect to overall biomass or community composition (Fig. 6B). The similarity of stations based on biomass composition was high, with the average similarity among all combinations of

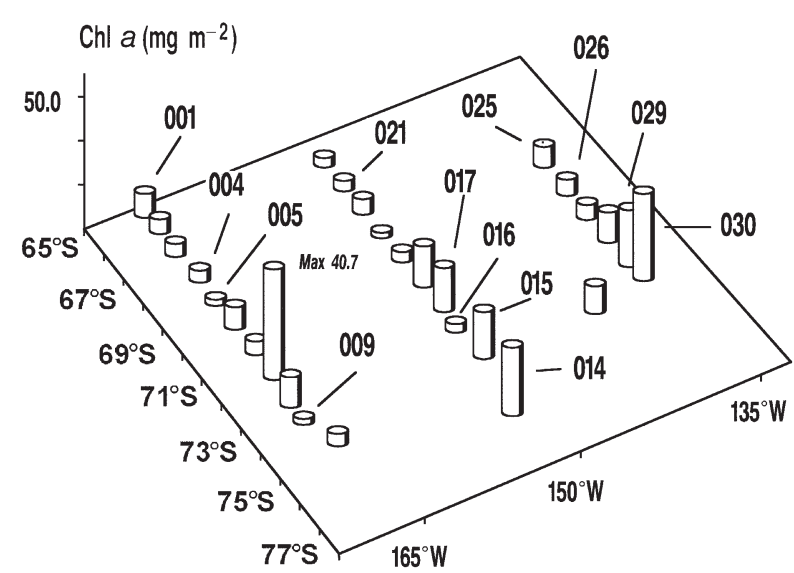

Fig. 5. Integrated chl a $\left(\mathrm{mg} \mathrm{m}^{-2}\right)$ in floes during NBP99-1. Stations analyzed for community structure are identified. See Fig. 1 for a more accurate station location

paired PSI comparisons for ice samples averaging $75 \%$ (range 40 to $96 \%$ ) and a similar comparison among the slush samples averaging $45 \%$ (range 3 to $90 \%$ ). All ice stations and several slush samples formed single-linkage clusters at $>70 \%$ similarity (clustering dendrogram is not shown but the MDS of the comparison is shown in Fig. 7). As suggested by the range of PSI values (above), the surface slush samples were considerably more variable than samples from within the ice. These formed distinct station-clusters or appeared as single-station outliers in the MDS plot (see Fig. 7).

Diatoms contributed most of the similarity to the clustering relationship among ice floes (Table 4). However, the diatoms were less important in determining relationships within the surface slush; Phaeocystis, other autotrophic flagellates, autotrophic dinoflagellates, ciliates, and heterotrophic flagellates were relatively more important (see Table 4). Clusters B and C (Fig. 7) were those where Phaeocystis dominated the assemblage biomass (Cluster B) or where Phaeocystis biomass was proportionately higher (Cluster C) (Table 4). Outlying surface slush samples 008 and 009 were dominated by Pyramimonas spp. (AFL), and sample 010 was one where autotrophic dinoflagellates Gymnodinium spp. predominated. Surface slush sample 027 was unusual in that a bloom of the ciliate Gymnozoum spp. dominated the assemblage. As was also the case for winter samples, a wide variety of diatom species was observed over the study area, and the dominance varied from sample to sample. Abundant and/or commonly occurring species included Entomoneis spp., Fragiliariopis (curta and/or cylindrus), Rhizosolenia spp., Chaetoceros neogracilis, Pseudo-nitzschia spp., Corethron, and Chaetoceros dichaeta. 

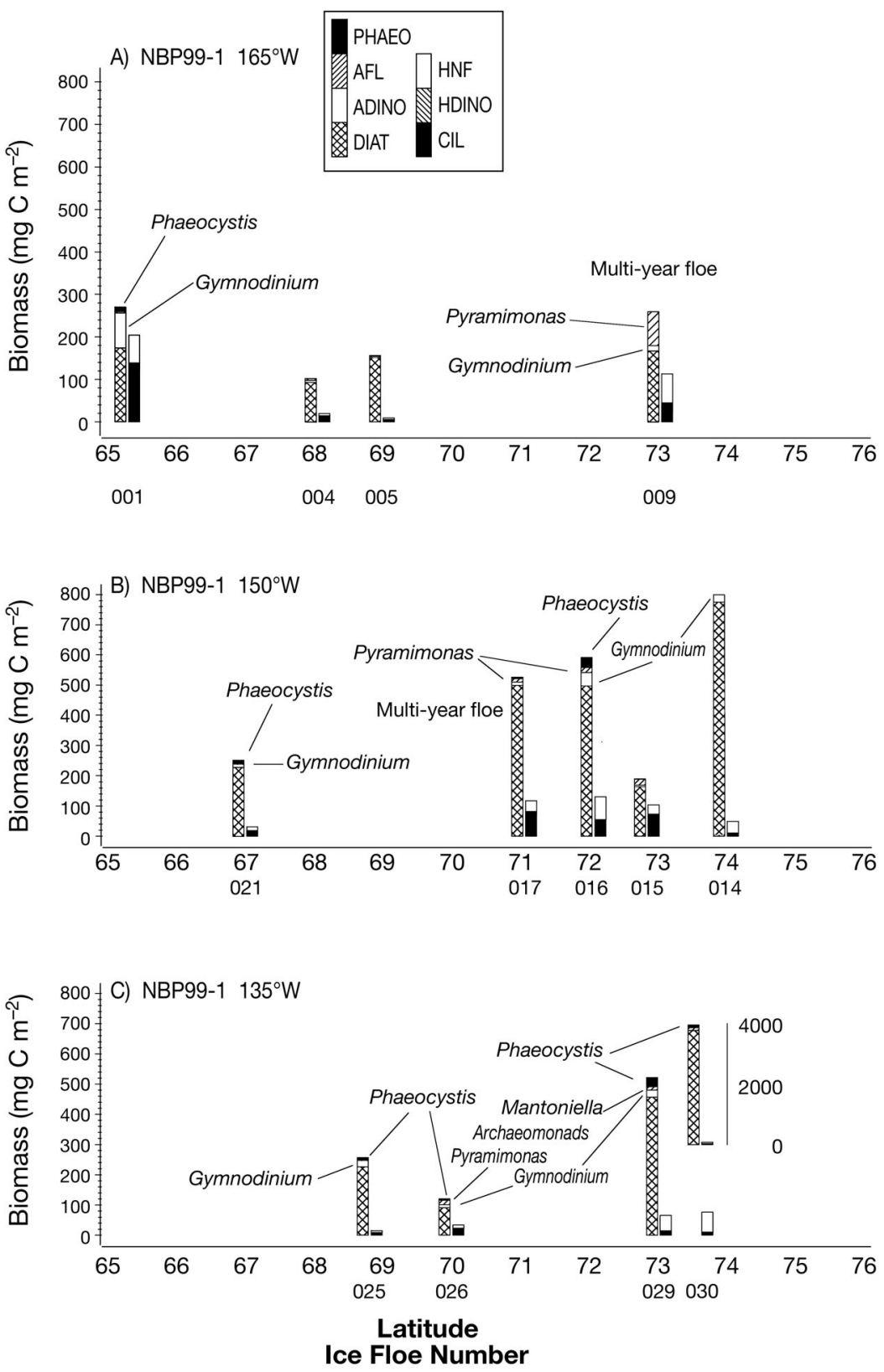

Fig. 6. Integrated biomass in floes sampled during NBP99-1. The first bar is autotrophic biomass; the second bar is heterotrophic biomass. (A) Transect along $165^{\circ} \mathrm{W}$. (B) Transect along $150^{\circ} \mathrm{W}$. (C) Transect along $135^{\circ} \mathrm{W}$. Note that data for autotrophic biomass are way off-scale at Stn 030 and are plotted above on a different scale. Heterotrophic biomass data for this station are best seen on the plot scaled 0 to $800 \mathrm{mg} \mathrm{C} \mathrm{m}^{-2}$. Prominent species are identified for some stations. See 'Discussion' for details and Table 1 for abbreviations

\section{DISCUSSION}

The overall objectives of the study were to examine the composition of ice assemblages in the Ross Sea region and to assess the pattern of seasonal development. NBP98-3 provided a snapshot of the autumn to winter transition across the region. NBP99-1 documented mid- to late-summer conditions across the same range of latitudes. The 2 cruises, however, do not provide an ideal comparison for the region because the autumn to winter cruise (NBP98-3) was located to the west of the summer cruise transects (NBP99-1; see Fig. 1). In particular, the autumn cruise is unlikely to provide a general model for autumn to winter community development across a latitudinal gradient that can be applied to other regions because we (Garrison et al. 2003) found that the development of communities in the Ross Sea Polynya was markedly affected by northward ice drift resulting from kabatic winds blowing off the Ross Ice Shelf (see also Arrigo et al. 2003). Thus, although by the end of the cruise light levels and temperatures were low throughout the study area and maximum autumn biomass accumulation should have been achieved, the northward advection of ice during its development is likely to have optimized growth in the oldest ice floes. Therefore, other ice-covered areas lacking northward ice displacement may show lower levels of autumn biomass accumulation and less pronounced spatial (latitudinal) variability (Garrison et al. 2003). Late-summer observations in the region of the autumn-to-winter cruise were not possible because the Ross Sea Polynya was largely ice-free by the time of our summer cruise.

Despite these differences in location and in the dynamics of ice formation and drift, seasonal differences were apparent, as most measures of aggregate biomass ( $\mathrm{chl}$ a) and the total carbon biomass showed 2- to 12-fold increases (either median or maximum values) during the summer as compared to autumn cruise values, depending on the biomass measure (Tables $1 \& 2$ ). Comparisons of the biomass and abundance of many species (Table 1) also showed similar (apparent) summer increases. However, statistical comparisons of the major groups using discrete-depth data indicated considerable overlap between values in autumn ice, summer ice, and surface slush; the only significantly higher biomass concentrations were for autotrophic flagellates 


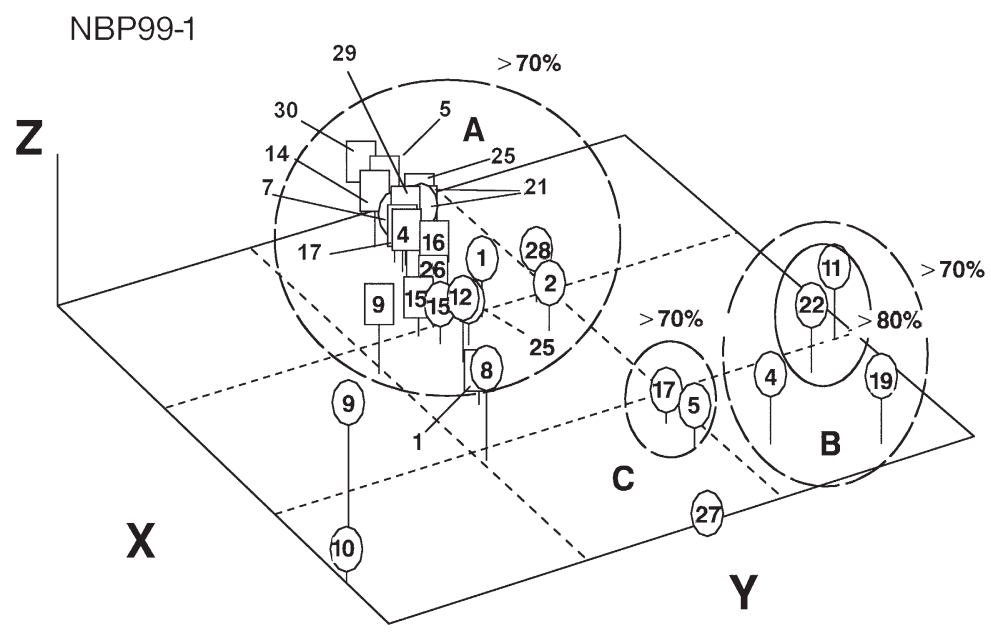

Fig. 7. Multidimensional scaling plots (MDS) of percent similarity matrix (PSI) based on the composition of the biomass. Rectangles: ice samples; ovals: surface slush samples. The large dashed circle indicates stations that clustered at the $70 \%$ similarity level. The large cluster (A) clustered with all stations shown in Fig. 4 at the $70 \%$ similarity level. Clusters B and C were also distinct at the $70 \%$ similarity level. Stns 8, 9, 10, and 27 were distinct from one another, and from clusters at similarities $>70 \%$

and Phaeocystis in surface slush (Duncan nonparametric multiple-range test, $\alpha=0.05$; SAS Institute 1989). It is not clear whether the January cruise (NBP99-1) represents Ross Sea ice communities at their maximum stage of development. Observations from near-shore fast ice areas suggest maximum production and biomass build-up occurs during the early summer in bottom layer communities (e.g. Watanabe et al. 1990, Arrigo et al. 1993). For example, Archer et al. (1996) noted an almost 2-fold decrease in total microbial biomass from mid-November through late December at a coastal land-fast study site. These authors attributed the observed decline to the loss of a rich bottom-layer assemblage, characteristic of coastal fast ice regions. Whereas bottom communities in drifting pack ice are thought to be different from those in landfast ice (Garrison et al. 1986), during the present study the highest summer biomass floe (Sample 030, 4 g C $\mathrm{m}^{-2}$ total microbial biomass) was largely made up of a diffuse bottom layer population, reaching a maximum in the bottom of the floe but extending well into the interior of the floe. Similarly, moderately high chl a levels were found in bottom layer assemblages during the autumn to winter cruise (NBP98-3) (Garrison et al. 2003). Ackley \& Sullivan (1994) have suggested the apparent lower development of bottom layer in pack ice can be attributed to snow cover and, thus, lower light levels at the ice-water interface, but we (Garrison et al. 2003) have argued that losses by melting at the undersides of floes may also be important. Even if bottom communities in the pack-ice regions of the Ross Sea do not reach the high biomass levels observed in landfast ice, the dynamics may be similar to those reported by Archer et al. (1996), with maximum development in late November and early December. The lack of samples from this period prevents us from evaluating this possibility.

It has also been observed in other regions of the pack ice that production and biomass build-up in offshore drifting pack ice apparently continues into the summer within surface layer assemblages (Burkholder \& Mandelli 1965, Dieckmann et al. 1998, Kristiansen \& Syvertsen 1990, Kristiansen et al. 1996). During our summer observations, the surface layer slush assemblages, although widely-distributed, had a relatively low biomass $(<1$ to $60.5 \mu \mathrm{g}$ chl $a^{-1}$ ). This estimate, however, may be conservative because we only included samples where we were able to take relatively large quantitative bulk samples of ice and brine. Other non-quantitative samples of interstitial water from slush (i.e. those where the interstitial water but not the suspended ice crystals were included) showed chl a levels of up to $1456 \mu \mathrm{g} \mathrm{l}^{-1}$ (Fritsen et al. 2001). High summer values have been reported for

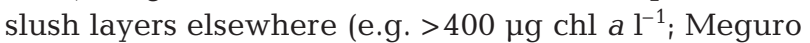
1962, Burkholder \& Mandelli 1965, Kristiansen \& Syvertsen 1990). Furthermore, modeling of surface populations in the Ross Sea over the late summer months (Arrigo et al. 1998) has suggested that integrated values of chl a in surface assemblages could reach $60 \mathrm{mg} \mathrm{m}^{-2}$ where sea ice persists. Thus, it seems

Table 4. Contribution of major groups to cluster similarity for summer samples. The individual contribution of major groups to the percent similarity analysis (PSI). The comparisons shown are among all core samples (ice floes), among all surface slush (slush) and within the clusters identified in Fig. 7. See Table 1 for abbreviations

\begin{tabular}{|lccccrr|}
\hline & $\begin{array}{c}\text { Ice floes } \\
(78)\end{array}$ & $\begin{array}{c}\text { Slush } \\
(153)\end{array}$ & $\begin{array}{c}\text { Cluster A } \\
(210)\end{array}$ & $\begin{array}{c}\text { Cluster B } \\
(6)\end{array}$ & $\begin{array}{c}\text { Cluster B' } \\
(1)\end{array}$ & $\begin{array}{c}\text { Cluster C } \\
(1)\end{array}$ \\
\hline & & & & & & \\
DIAT & 0.80 & 0.35 & 0.77 & 0.11 & 0.23 & 0.19 \\
CIL & 0.07 & 0.18 & 0.08 & 0.06 & 0.01 & 0.38 \\
HNF & 0.05 & 0.11 & 0.05 & $<0.01$ & $<0.01$ & 0.14 \\
ADINO & 0.03 & 0.11 & 0.04 & 0.06 & 0.04 & 0.02 \\
AFL & 0.02 & 0.06 & 0.03 & 0.01 & $<0.01$ & 0.01 \\
PHAEO & 0.01 & 0.15 & 0.02 & 0.72 & 0.70 & 0.25 \\
HDINO & $<0.01$ & 0.02 & 0.01 & 0.02 & 0.02 & $<0.01$ \\
\hline
\end{tabular}


likely that development of surface communities would have continued in the ice fields remaining after our observations (i.e. into February and March). Moreover, the study area during NBP99-1 was in an area where ice persists throughout the summer (see Fig. 2 in Garrison et al. 2003), and in a similar area of persistent ice in the Amundsen Sea, Thomas et al. (1998) describe high

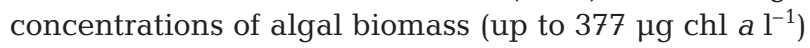
in the interior assemblages of decomposing ice floes in February and March. Such late season development of internal ice communities in the eastern Ross Sea would also have been missed by our sampling program.

One of the surprising findings of the study was the consistency in composition of the major biomass components within ice floes over a wide range of biomass values (Table 2, Figs. 4 \& 7). Most samples from within the ice floes from both seasons cluster at about the $70 \%$ PSI level. Minor differences (clusters formed at similarity of around $80 \%$ PSI) were apparent within the autumn cruise based on variations among diatoms, autotrophic dinoflagellates, and ciliates (Table 3). Thus, the overall dynamics within the ice community may be determined by relatively few taxa adapted to grow and survive within the ice habitat. Gleitz et al. (1998), in a study focused only on the diatom component, have suggested such a pattern of community dynamics. Similarly surprising was that there was no statistical relationship with ice structural type; that is, values for the biomass of the major groups compared among frazil, congelation, infiltration, platelet, and mixed structural types were not significantly different (Duncan multiple-range test; $\alpha=0.05$ ). With respect to vertical distributions within ice floes, diatom biomass was statistically higher in the bottom layer than the surface, but values from within the interior of floes overlapped with those of both the bottom and surface layers. None of the other groups showed vertical patterns that were significant.

The most divergent samples were among the summer surface-slush layer blooms where single species often predominated in low diversity assemblages (e.g. blooms dominated by Phaeocystis, Pyramimonas spp., Gymnodinium spp. and the ciliate Gymnozoum sp.). This striking variability may be related to events that lead to the formation of the surface blooms. It is not known whether surface blooms develop from a seed population from within the ice or whether cells are introduced from the water column by flooding at the snow-ice interface (Garrison \& Buck 1991, Garrison \& Mathot 1996). On the one hand, the blooms of taxa such as Phaeocystis (Garrison et al. 1991), Pyramimonas (Daugbjerg et al. 2000), and Pseudopleurochloris (Moro et al. 2000), within surface populations would be consistent with the opportunistic seeding during flooding events. On the other hand, some surface slush blooms are dominated by taxa common within ice floes during the autumn and winter. The presence of dinoflagellate cysts and chrysophyte statocysts (Buck et al. 1992, Stoecker et al. 1992, 1993) has suggested life-cycle strategies that would allow surface blooms to develop from populations overwintering within the ice. The presence of a 'Gymnodinium-like' bloom in 1 surface slush bloom and the finding of this form (including cysts) as a common form in autumn ice samples may be an example of a surface bloom initiated from resting stages within the ice (also see Stoecker et al. 1992).

Over 120 taxa were recognized within the ice (Table 1), and it is clear that grouping organisms into higher taxonomic categories must obscure the actual diversity within the ice. Similarly, analysis of integrated data for floes would blur the distinction of microhabitats and any assemblages that might be associated with them. Nevertheless, because there are few studies where the biota have been identified and because the ability to discern taxa at the species level differs markedly among the groups present, simplification in the initial stages of the community analysis was necessary. Further work is under way to determine whether more specific seasonal or spatial relationships can be resolved within the sea-ice environments, with analyses focused on species or at least at lower taxonomic groupings. In some ways, the high level of similarity of community structure across a large ice-covered region and the predominance of species common in both ice and water (Table 1) suggests that sea ice does not constitute a unique habitat in the Antarctic pelagic system.

Acknowledgements. This study was supported by NSF grants to D.L.G. and M.M.G. (OPP 9614201), M.O.J and S.L. (OPP 9614844), and C.H.F. (OPP-9814972 and OPP9910098). We thank J. Ikävalko, D. Neenan, C. Venn, J. L. Tison, J. M. Rintala, H. J. Marchant, W. Petz and N. Daugbjerg for various assistance during the field programs. We thank H. Marchant and W. Petz for help with some of the identifications. We also thank the Captain and crew of the 'Nathaniel B. Palmer' for their assistance, and we give special thanks to J. Barnes and other Antarctic Support Associates staff for their technical and field support. Interpretations and conclusions are those of the authors and do not imply the endorsement of the National Science Foundation.

\section{LITERATURE CITED}

Ackley SF, Sullivan CW (1994) Physical controls on the development and characteristics of Antarctic sea ice biological communities - a review and synthesis. Deep-Sea Res I 41:1583-1604

Archer SD, Leakey RJG, Burkill PH, Sleigh MA, Appleby CJ (1996) Microbial ecology of sea ice at a coastal Antarctic site: community composition, biomass and temporal change. Mar Ecol Prog Ser 135:179-195

Arrigo KR, Kremer JN, Sullivan CW (1993) A simulated Antarctic fast ice ecosystem. J Geophys Res 98:6929-6946 
Arrigo K, Worthen DL, Dixon P, Lizotte MP (1998) Primary productivity of near surface communities within Antarctic pack ice. In: Lizotte MP, Arrigo KR (eds) Antarctic sea ice: biological processes, interactions and variability. Antarctic Research Series 73. American Geophysical Union, Washington, DC, p 23-43

Arrigo KR, Robinson DH, Dunbar RB, Leventer AR, Lizotte MP (2003) Physical controls of chlorophyll a, POC, and TNP distributions in the pack ice of the Ross Sea, Antarctica. J Geophys Res 108:3316-3338

Buck KR, Thomsen HA, Marchant HJ, Garrison DL (1990a) Kakoeca antarctica gen. et sp. nov., a loricate choanoflagellate (Acanthoecidae, Choanoflagellida), from Antarctic sea ice with a unique protoplast suspensory membrane. Zool Scr 19:389-394

Buck KR, Bolt PA, Garrison DL (1990b) Phagotrophy and fecal pellet production by an athecate dinoflagellate in Antarctic sea ice. Mar Ecol Prog Ser 60:75-84

Buck KR, Bolt PA, Betham WN, Garrison, DL (1992) A dinoflagellate cyst from Antarctic sea ice. J Phycol 28:15-18

Burkholder PR, Mandelli EF (1965) Productivity of microalgae in Antarctic sea ice. Science 148:872-874

Coleman AW (1980) Enhanced staining of bacteria in natural environments by fluorochrome staining of DNA. Limnol Oceanogr 25:948-951

Corliss JO, Snyder RA (1986) A preliminary description of several new ciliates from the Antarctic, including Cohnilembus grassei n. sp. Protistologica 22:39-46

Dahms HU, Dieckmann GS (1987) Drescheriella glacialis gen. nov., sp. nov. (Copepoda, Harpacticoida) from Antarctic sea ice. Polar Biol 7:329-337

Daly KL (1990) Overwintering development, growth and feeding of larval Euphausia superba in the Antarctic marginal ice zone. Limnol Oceanogr 37:1564-1576

Daugbjerg N, Marchant JH, Thomsen HA (2000) Life history stages of Pyramimonas tychotreta (Prasinophyceae, Chlorophyta), a marine flagellate from the Ross Sea, Antarctica. Phycol Res 48:199-209

Dieckmann GS, Eicken H, Haas C, Garrison DL and 7 others (1998) A compilation of data on sea ice algal standing crop from the Bellinghausen, Admundsen and Weddell Seas from 1983-1994. In: Lizotte MP, Arrigo KR (eds) Antarctic sea ice: biological processes, interactions and variability. Antarctic Research Series 73, American Geophysical Union, Washington, DC, p 85-92

Eppley RW, Reid FMH, Strickland JDH (1970) Estimates of phytoplankton crop size, growth rate, and primary production. In: Strickland JDH (ed) The ecology of the plankton off La Jolla California in the period April through September 1967. Bull Scripps Inst Oceanogr Univ Calif 17:33-42

Field JG, Clarke KR, Warwick RM (1982) A practical strategy for analyzing multispecies distribution patterns. Mar Ecol Prog Ser 8:37-52

Fritsen CH, Coale S, Neenan D, Gibson A, Garrison D. (2001) Biomass, production and microhabitat characteristics near the freeboard of ice floes in the Ross Sea during the austral summer. Ann Glaciol 33:280-286

Garrison DL (1991) Antarctic sea ice biota. Am Zool 31:17-33

Garrison DL, Buck KR (1985) Sea ice algal communities in the Weddell Sea: species composition in ice and planktonic assemblages. In: Gray JS, Christiansen ME (eds) Proc 18th European Mar Biol Symp. Biology of polar regions and effects of stress on marine organisms. John Wiley, New York, p 103-122

Garrison DL, Buck KR (1986) Organism losses during ice melting: a serious bias in sea ice community studies. Polar Biol 6:237-239
Garrison DL, Buck KR (1989) The biota of Antarctic pack ice in the Weddell Sea and Antarctic Peninsula regions. Polar Biol 10:211-219

Garrison DL, Buck KR (1991) Surface-layer sea ice assemblages in Antarctic pack ice during the austral spring: environmental conditions, primary production and community structure. Mar Ecol Prog Ser 75:161-172

Garrison DL, Close AR (1993) Winter ecology of the sea ice biota in Weddell Sea pack ice. Mar Ecol Prog Ser 96:17-31

Garrison DL, Mathot S (1996) Pelagic and sea ice microbial communities. In: Ross RM, Hofmann EE, Quetin LB (eds) Foundations for ecological research west of the Antarctic Peninsula. Antarctic Research Series 70, American Geophysical Union, Washington, DC, p 155-172

Garrison DL, Sullivan CW, Ackely SF (1986) Sea ice microbial communities in Antarctica. BioScience 36:243-250

Garrison DL, Buck KR, Fryxell, GA (1987) Algal assemblages in antarctic pack ice and in ice-edge plankton. J Phycol 23:564-572

Garrison DL, Gowing MM, Hughes MP (1998) Nano- and microplankton in the northern Arabian Sea during the Southwest Monsoon, August-September 1995 A USJGOFS study. Deep-Sea Res II 45:2269-2299

Garrison DL, Jeffries MO, Gibson A, Coale SL, Neenan D, Fritsen C, Okolodkov YB, Gowing MM (2003) Development of sea ice microbial communities during autumn ice formation in the Ross Sea. Mar Ecol Prog Ser 259:1-15

Gast RJ, Beaudoin D, Dennett MR, Moran DM, Caron DA (2001) Genetic diversity of protists in antarctic marine environments. Annu Meeting Am Soc Limnol Oceanogr, Feb 2001, Albuquerque. American Society of Limnology and Oceanography, Waco, TX, p 57

Gast RJ, Caron DA, Moran DM, Rose JM, Dennett MR, Schaeff RA, Patterson DJ (2002) Protistan molecular ecology and physiology in Antarctic waters, LExEn and Microbial Observatory Workshop, Sep 22-24, 2002, Arlington. US National Science Foundation, Arlington, VA, p 34

Gast RJ, Moran DM, Dennett MR, Rose JM, Caron DA (2003) Linking protistan genotype, morphology and physiology from ice and water samples of the Ross Sea, Antarctica. Annu Meeting Am Soc Limnol Oceanogr, Feb 2003, Salt Lake City. American Society of Limnology and Oceanography, Waco, TX, p 57

Gleitz M, Bartsch A, Dieckmann G, Eicken H (1998) Composition and succession of sea ice diatom assemblages in the eastern and southern Weddell Sea, Antarctica. In: Lizotte MP, Arrigo KR (eds) Antarctic sea ice: biological processes, interactions and variability. Antarctic Research Series 73. American Geophysical Union, Washington, DC, p 107-120

Gowing MM (2003) Large viruses and infected microeukaryotes in Ross Sea summer pack ice habitats. Mar Biol 142: 1029-1040

Gowing MM, Riggs BE, Garrison DL, Gibson AH, Jeffries MO (2002) Large viruses in Ross Sea autumn pack ice habitats. Mar Ecol Prog Ser 241:1-11

Gowing MM, Garrison DL, Gibson AH, Krupp JM, Jeffries, MO, Fritsen, CH (2004) Bacterial and viral abundance in Ross Sea summer pack ice communities. Mar Ecol Prog Ser 279:3-12

Hobbie JE, Daley RJ, Jasper S (1977) Use of Nucleopore filters for counting bacteria by fluorescence microscopy. Appl Environ Microb 33:1225-1228

Horner R (1985a) Ecology of sea ice microalgae. In: Horner R (ed) Sea ice biota. CRC Press, Boca Raton, FL, p 83-103

Horner R (1985b) Taxonomy of sea ice microalgae In: Horner R (ed) Sea Ice Biota, CRC Press, Boca Raton, FL, p 148-157 
Jeffries MO, Krouse HR, Hurst-Cushing B, Maksym T (2001) Snow ice accretion and snow cover depletion on Antarctic first-year sea ice floes. Ann Glaciol 33:51-60

Kristiansen S, Syvertsen EE (1990) Sea ice algae in the Weddell Sea during austral spring. EOS 71:79

Kristiansen S, Farbrot T, Kuosa H, Myklestad S, von Quillfeldt $\mathrm{CH}$ (1996) Nitrogen uptake in the infiltration community, an ice algal community in Antarctic pack ice. Polar Biol 19:307-315

Laurion I, Demers S, Vezina AF (1995) The microbial food web associated with the algal assemblage: biomass and bacterivory of nanoflagellate protozoans in Resolute Passage (High Canadian Arctic). Mar Ecol Prog Ser 120:77-87

Legendre L, Ackley SF, Dieckmann GS, Gulliksen B and 6 others (1992) Ecology of sea ice biota 2. global significance. Polar Biol 12:429-444

Lizotte MP (2001) The contributions of sea ice algae to Antarctic marine primary production. Am Zool 41:57-73

Maranger R, Bird DF, Juniper SK (1994) Viral and bacterial dynamics in Arctic sea ice during the spring algal bloom near Resolute, N.W.T., Canada. Mar Ecol Prog Ser 111:121-127

Marchant HJ, Buck KR, Garrison DL, Thomsen HA (1989) Mantoniella in Antarctic waters including a description of M. antarctica. J Phycol 25:167-174

Mathot S, Smith WO, Carlson CA, Garrison DL, Gowing MM, Vickers CL (2000) Carbon partitioning within Phaeocystis antarctica (Prymnesiophyceae) colonies in the Ross Sea, Antarctica. J Phycol 36:1049-1056

Meguro H (1962) Plankton ice in the Antarctic Ocean. Antarctic Record (Tokyo) 14:72-79

Mitchell JG, Silver MW (1982) Modern archaeomonads indicate sea-ice environments. Nature 296:437-443

Montresor M, Procaccini G, Stoecker DK (1999) Polarella glacialis gen. nov., sp. nov. (Dinophyceae): Suessiaceae are still alive. Polar Biol 35:186-197

Montresor M, Lovejoy C, Orsini L, Procaccini G (2003) Bipolar distribution of the cyst-forming dinoflagellate Polarella glacialis. Polar Biol 26:186-194

Moro I, Paccagnella R, Barbante C, Andreoli C (2000) Microalgal communities of the sea ice, ice-covered and ice-free waters of Wood Bay (Ross Sea, Antarctica) during the austral summer 1993-94. PSZN I Mar Ecol 21:233-245

Palmisano AC, Garrison DL (1993) Microorganisms in Antarctic sea ice. In: Friedmann EI (ed) Antarctic microbiology. Wiley-Liss, New York, p 167-218

Parsons,TR, Maita Y, Lalli, CM (1984) A manual of chemical and biological methods for seawater analysis. Pergamon Press, Oxford

Putt M, Stoecker DK (1989) An experimentally determined carbon:volume ratio for marine "oligotrichous" ciliates from estuaries and coastal waters. Limnol Oceanogr 34:1097-1103

Editorial responsibility: Otto Kinne (Editor-in-Chief), Oldendorf/Luhe, Germany
SAS Institute (1989) SAS/STAT Users Guide, Version 6, 4th edn, Vol 2. SAS Institute, Cary, NC

Sime-Ngando T, Juniper SK, Demers S (1997) Ice-brine and planktonic microheterotrophs from Saroma-ko Lagoon, Hokkaido (Japan): quantitative importance and trophodynamics. J Mar Syst 11:149-161

Sime-Ngando T, Demers S, Juniper SK (1999) Protozoan bacterivory in the ice and the water column of a cold temperate lagoon. Microb Ecol 37:95-106

Stewart FJ, Fritsen CH, Garrison DL, Gibson AH (2001) Bacteria-algae associations in the sea ice and upper water column of the Ross Sea in the Late Austral Summer. Am Soc Limnol Oceanogr 2001 Aquat Sci Meeting, Feb 12-16, 2001. Abstract Vol, Albuquerque. American Society of Limnology and Oceanography, Waco, TX, p 134

Stoecker DK, Buck KR, Putt M (1992) Changes in the sea-ice brine community during the spring-summer transition, McMurdo Sound, Antarctica. I. Photosynthetic protists. Mar Ecol Prog Ser 84:265-278

Stoecker DK, Buck KR, Putt M (1993) Changes in the sea-ice brine community during the spring-summer transition, McMurdo Sound, Antarctica. II. Phagotrophic protists. Mar Ecol Prog Ser 95:103-113

Thomas DN, Lara RJ, Haas C, Schnarck-Shiel, SB, Dieckmann GS, Kattner, G, Nöthig, EM, Mizdalski E (1998) Biological soup within decaying summer sea ice in the Amundsen Sea, Antarctica. In: Lizotte MP, Arrigo KR (eds) Antarctic sea ice: biological processes, interactions and variability. Antarctic Research Series 73, American Geophysical Union, Washington, DC, p 161-171

Thomsen HA, Buck KR, Coale SL, Garrison DL, and Gowing MM (1990) Loricate choanoflagellates (Acanthoecidae, Choanoflagellida) from the Weddell Sea, Antarctica. Zool Scr 19:367-387

Thomsen HA, Buck KR, Bolt PA, Garrison DL (1991) Fine structure and biology of Cryothecomonas gen nov (Protista Incertae Sedis) from the ice biota. Can J Zool 69:1048-1070

Thomsen HA, Garrison DL, Kosman (1997) Choanoflagellates (Acanthoecidae, Choanoflagellida) from the Weddell Sea, Antarctica, taxonomy and community structure with particular emphasis on the ice biota; with preliminary remarks on choanoflagellates from Arctic sea ice (Northeast Water Polynya, Greenland). Arch Protistenkd 148:77-114

Washington HG (1984) Diversity, biotic and similarity indices: a review with special relevance to aquatic ecosystems. Water Res 18:653-694

Watanabe K, Satoh H, Hoshiai T (1990) Seasonal variation in ice algal assemblages in the fast ice near Syowa Station in 1983/84. In: Kerry KR, Hempel G (eds) Antarctic ecosystems. Ecological change and conservation. SpringerVerlag, Berlin, Heidelberg, p 136-142

Submitted: October 18, 2004; Accepted: March 22, 2005

Proofs received from author(s): August 2, 2005 